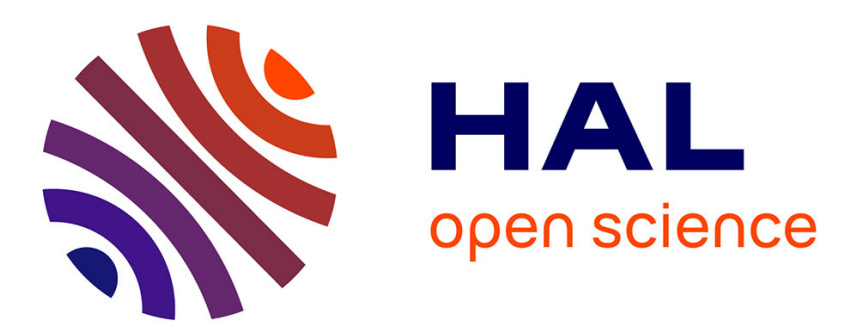

\title{
A high order moment method simulating evaporation and advection of a polydisperse liquid spray
}

Damien Kah, Frédérique Laurent, Marc Massot, Stéphane Jay

\section{To cite this version:}

Damien Kah, Frédérique Laurent, Marc Massot, Stéphane Jay. A high order moment method simulating evaporation and advection of a polydisperse liquid spray. Journal of Computational Physics, 2012, 231 (2), pp.394-422. 10.1016/j.jcp.2011.08.032 . hal-00536512v2

\section{HAL Id: hal-00536512 \\ https://hal.science/hal-00536512v2}

Submitted on 6 Sep 2011

HAL is a multi-disciplinary open access archive for the deposit and dissemination of scientific research documents, whether they are published or not. The documents may come from teaching and research institutions in France or abroad, or from public or private research centers.
L'archive ouverte pluridisciplinaire HAL, est destinée au dépôt et à la diffusion de documents scientifiques de niveau recherche, publiés ou non, émanant des établissements d'enseignement et de recherche français ou étrangers, des laboratoires publics ou privés. 


\title{
A high order moment method simulating evaporation and advection of a polydisperse liquid spray
}

\author{
D. Kah \\ CNRS, UPR 288, Laboratoire EM2C, Grande Voie des Vignes, 92290 Châtenay-Malabry, France \\ Ecole Centrale Paris, Grande Voie des Vignes, 92295 Chatenay-Malabry Cedex, France \\ IFP - Energies nouvelles, 1 et 4 avenue de Bois Préau 92852 Rueil-Malmaison, France \\ F. Laurent, M. Massot* \\ CNRS, UPR 288, Laboratoire EM2C, Grande Voie des Vignes, 92290 Châtenay-Malabry, France \\ Ecole Centrale Paris, Grande Voie des Vignes, 92295 Chatenay-Malabry Cedex, France \\ S. Jay \\ IFP - Energies nouvelles, 1 et 4 avenue de Bois Préau 92852 Rueil-Malmaison, France
}

\begin{abstract}
In this paper, we tackle the modeling and numerical simulation of sprays and aerosols, that is dilute gasdroplet flows for which polydispersity description is of paramount importance. Starting from a kinetic description for point particles experiencing transport either at the carrier phase velocity for aerosols or at their own velocity for sprays as well as evaporation, we focus on an Eulerian high order moment method in size and consider a system of partial differential equations (PDEs) on a vector of successive integer size moments of order 0 to $N, N>2$, over a compact size interval. There exists a stumbling block for the usual approaches using high order moment methods resolved with high order finite volume methods: the transport algorithm does not preserve the moment space. Indeed, reconstruction of moments by polynomials inside computational cells coupled to the evolution algorithm can create $N$-dimensional vectors which fail to be moment vectors: it is impossible to find a size distribution for which there are the moments. We thus propose a new approach as well as an algorithm which is second order in space and time with very limited numerical diffusion and allows to accurately describe the advection process and naturally preserves the moment space. The algorithm also leads to a natural coupling with a recently designed algorithm for evaporation which also preserves the moment space; thus polydispersity is accounted for in the evaporation and advection process, very accurately and at a very reasonable computational cost. These modeling and algorithmic tools are referred to as the EMSM (Eulerian Multi Size Moment) model. We show that such an approach is very competitive compared to multi-fluid approaches, where the size phase space is discretized into several sections and low order moment methods are used in each section, as well as with other existing high order moment methods. An accuracy study assesses the order of the method as well as the low level of numerical diffusion on structured meshes. Whereas the extension to unstructured meshes is provided, we focus in this paper on cartesian meshes and two 2D test-cases are presented: Taylor-Green vortices and turbulent free jets, where the accuracy and efficiency of the approach are assessed.
\end{abstract}

Key words: Polydisperse sprays, aerosols, high order moment method, moment space, canonical moments, Eulerian multi-fluid model, Maximum Entropy reconstruction, kinetic finite volume schemes 2000 MSC: 35Q35, 65M12, 65M99, 76T10

\footnotetext{
This work was supported by a Young Investigator Award for M. Massot (ANR-05-JCJC-0013 - jéDYS - 2005-2009) from ANR in France (National Research Agency) and by a CNRS financial support (PEPS "Projet Exploratoire Pluridisciplinaire" 2007-2009, from the ST2I and MPPU Departments of CNRS, coordination: A. Bourdon and F. Laurent) .

* Corresponding author - marc.massot@em2c.ecp.fr - Present address: Center for Turbulence Research, Stanford University, Building 500, Stanford CA 94305, mmassot@stanford.edu
} 


\section{Introduction}

The study of multi-phase flow constituted of a disperse cloud of liquid droplets carried by a gaseous flow field, also called sprays or aerosols depending on whether they follow the gaseous flow field or have their own dynamics, is of major importance and interest in many fields such as combustion, chemical engineering [42], rocket booster propulsion [13,14] and atmospheric studies [50]. A key example involving this kind of flow is fuel injection in internal combustion engines. The fuel is typically stored as a liquid phase and injected at high pressures into a gas-filled combustion chamber. Due to the interactions with the surrounding gas, the dense liquid core atomizes and eventually results in a dilute polydisperse spray, in a region located downstream of the injector and where combustion takes place. The spatial topology of the fuel mass fraction is a direct consequence of the turbulent dispersion and evaporation of the spray in that region and is strongly related to size distribution since droplet dynamics and evaporation are conditioned on droplet size. The spray dynamics thus governs the resulting combustion regimes, and therefore has a strong influence on the key undesirable by-products of an engine, such as pollutants and soots. Since experimental measurements of various combustion chamber configurations for design purposes can be very expensive, computational models for multi-phase flows have recently become widely studied. Therefore, it is of great interest to design reliable models for such polydisperse sprays flow, as well as numerical methods which are as accurate as possible, but also tractable for large scale computations on parallel architectures. It is for this purpose that the scope of this work is the introduction of a new numerical approach based on high order size moment methods and on a rigorous mathematical background for dilute particulate flow regions which is both stable and computationally efficient.

Dilute gas-droplet flows are typically described by a kinetic equation modeling clouds of point particles, represented by its number density function (NDF), through transport, droplet-fluid interactions, and eventually droplet-droplet interactions in the framework of moderately dense cases. In most cases the sprays are polydisperse and experience size evolution due to evaporation; in such configurations, typical of reactive flows, the description of the size distribution is of paramount importance. The Knudsen number is assumed to be high so that the collision between droplets can be neglected. Additionally, the droplet Stokes number based on some typical gas flow time can range from very small Stokes numbers (aerosols) to Stokes numbers of the order of one (sprays). The principal modeling challenge is the simulation of polydispersity and dynamics of the droplet population. Therefore, in this contribution, we will consider one-way coupling for comparison purposes, i.e. we will assume that the fluid velocity is given and influences the spray/aerosol dynamics and evaporation. Nevertheless, the extension to two-way coupling is straightforward and all the easier since the two phases are described by an Eulerian model.

Since the direct resolution of the kinetic equation is often intractable due to the large number of independent variables, stochastic Lagrangian methods "discretize" the NDF into "parcels", the dynamics of which is integrated. This approach has been widely used and has shown to be efficient in numerous cases (see example [19] and references herein). While quite accurate, its main drawback is the coupling of an Eulerian description for the gaseous phase to a Lagrangian description of the disperse phase, thus offering limited possibilities of vectorization/parallelization. Besides, as in any statistical approach, Lagrangian methods require a relatively large number of parcels to control statistical noise, and thus are computationally expensive, especially for polydisperse unsteady flows. An alternative to the Lagrangian approach is an Eulerian moment method. The closure of the velocity moments conditioned on size is classical and conducted through a usual hydrodynamic limit leading to an equilibrium velocity distribution, i.e. Maxwell-Boltzmann distribution up to zero temperature in the framework of direct numerical simulation $[32,8,27]$. Once a closure has been chosen in terms of velocity moments conditioned on droplet size, there are two options available for capturing the dynamics in the size phase space. One can either rely on size phase space discretization, with low order size moments in each section, as done in the multi-fluid approach developed in $[10,8,32]$ from [25]. The multi-fluid model considers only one size moment which accounts for the liquid mass density on small intervals of the size phase space called sections. Formally, the disperse phase is composed of several fluids exchanging mass and momentum with each other and with the gas through evaporation. This model has shown to yield simple transport algorithms for transport in physical space in $[10,9,8]$ implemented on parallel architectures $[20,22]$. However, the cost of 
the discretization in size phase space is high and still results in numerical diffusion since the method is first order in size discretization width [31]. Therefore, in terms of computational cost, the possibility of high order moment method considering a single size section is attractive.

At present, several moment methods have been designed. The first one consists of solving the evolution of moments of a presumed NDF (assumed as a log-normal law) [41]. Presumably, this is very interesting since knowing a priori, the profile of the NDF makes its reconstruction from the moments much easier. However, this assumption is restrictive in terms of the coverage of the physical processes. Moreover, this approach leads to serious numerical instabilities thus preventing its use for the treatment of an evaporating spray, since during the computation, a log-normal distribution function might not be reconstructed from the moment set dynamics. Another solution is high order moment methods, either quadrature method of moment (QMOM) where the dynamics of moments are evaluated after closing the source terms using quadrature methods (see [38] for example and references therein), or Direct Quadrature Method of Moment (DQMOM) [34] wherein equations are directly written on the quadrature weights and abscissas which describe the reconstructed distribution function having the same moments [19]. Such methods have proved to be very efficient in a number of configurations, such as agglomeration, sintering, coagulation-fragmentation... However, they encounter two sets of difficulties. First, they are not able to accurately predict the evaporating flux in general (see [19] for a precise statement) since they fail to reproduce the number density disappearing flux at zero droplet size, which is a point-wise value to be reconstructed from the set of moments. Consequently, further studies have been undertaken and have lead to a proper treatment of the evaporation term. The high order moment method together with the associated numerical scheme presented in [37] is a breakthrough in terms of accuracy, and can be effectively used for quantitative predictions of an evaporating spray. The second problem is related to transport in physical space. There exists a stumbling block for the usual approaches using high order moment method resolved with at least second order finite volume method: the transport algorithm does not preserve the moment space, that is, spatial reconstruction of moments by polynomials inside computational cells coupled to the evolution algorithm can create $N$-dimensional vectors which fail to be moment vectors: it is impossible to find a size distribution for which there are the moments. In fact two difficulties arise. The first one concerns the reconstruction of the moments in order to keep the integrity of the moment set. It has been seen that for a high order scheme, an independent spatial reconstruction of each moment does not insure that the moment space is preserved [50,38]. Besides, a second difficulty concerns the computation of the fluxes from the reconstructed quantities. For basic explicit time intergrators such as Explicit Runge-Kutta methods, the fluxes computation will introduce truncature errors for non constant reconstructions, and the preservation of the moment space will not be guaranteed any more, even if there were no problem in the reconstruction step. To achieve still a robust scheme, one possibility is to use projections onto the moment space. But this requires a number of numerical tests that may substantially impact the accuracy of the method, since the moments are artificially modified, and may increase the CPU time. A more recent realizable high order scheme for advection relying on QMOM has recently been proposed and evaluated in [48]. However, such an approach relies on the reconstruction of the quadrature weights whereas the quadrature abscissas are still treated in a first order manner.

Thus, in this contribution, we provide a new method for the transport in physical space of droplet size distributions using high order moment method in the size dimension, where the droplet size spans a compact interval, in the context of finite volume methods. We aim at transporting a vector of integer and successive size moment up to order $N, N>2$ in physical space and since we deal with sprays, we want to naturally couple it with an algorithm for evaporation. We thus propose a new approach as well as an algorithm which is second order in space and time with low numerical diffusion which allows to accurately describe the advection process and naturally guarantees that the vector always belongs to the moment space if the initial solution itself belongs to the moment space. We tackle both aerosols which are transported by the velocity of the carrier flow as well as more inertial droplets which have their own velocity field. The main algorithm is presented on cartesian meshes but the extension to unstructured meshes is provided. The required ingredients are a reconstruction of the independent canonical moments, which are well-suited since there are transported quantities, as well as the use of a time solver based on an exact resolution of the PDE in time, leading to the fact that the fluxes introduce no truncature error with respect to the spatial reconstruction. An exact computation of the fluxes is obtained using the fact that the equations on moments can be derived from a kinetic equation as originally proposed 
in Bouchut [3]. By coupling this approach to a recently designed algorithm for evaporation which also preserves the moment space [37], polydispersity is accounted for in the evaporation and advection process, very accurately and at a very reasonable computational cost. These modeling and algorithmic tools are referred to as the EMSM (Eulerian Multi Size Moment) model.

We show that such an approach is very competitive in terms of both computational efficiency and method accuracy compared to multi-fluid approches where the size phase space is discretized into several sections and low order moment methods are used in each section. A detailed accuracy and order study allows to demonstrate the proper order behavior of the method and we even compare the precision level to the method introduced in [48]. Two 2D test-cases are presented: Taylor-Green vortices and turbulent free jets. We thus show the accuracy and computational efficiency achievements of the present method and discuss its ability to be extended to more complex configurations as well as on massively parallel architectures in the conclusion.

The remainder of the paper is organized as follows. In Section 2, the kinetic equation for both aerosols and sprays are presented and the system of PDEs for high order size moment in the two cases obtained; furthermore, the main properties of the moment space as well as canonical moments are introduced as well as the main mathematical properties of the systems of PDEs which will be useful in order to design the new transport algorithm. Section 3 contains the development of the kinetic-based finite volume numerical scheme for the transport in physical space, wherein reconstruction on canonical moments are used for the computation of the fluxes. In Section 4 the essential points of the numerical scheme for the treatment of the evaporation term are highlighted. Section 5 is the results section; it covers both validation, detailed accuracy study as well as computational and accuracy evaluation of the numerical method and algorithm as well as its implementation for an evaporating polydisperse spray in 2D more complex configurations. Two 2D configurations of the gaseous flow field are considered, a Taylor Green steady and an unsteady weakly turbulent free jet. It allows us to compare the newly designed algorithm to the multi-fluid model in terms of accuracy and computational cost. A description of the extension of the method to unstructured meshes is presented in Appendix 7.C and the order and accuracy comparison with the method introduced in [48], in Appendix 7.B.

\section{High order moment models and associated properties}

This section is dedicated to the presentation of the system of PDEs modeling droplet size and velocity moments derived from the kinetic equation governing the evolution of the NDF. The cases of aerosols and sprays are treated separately as they lead to different systems of PDEs, even if they rely on the same basis at the kinetic level. Since we consider successive integer order size moments of the NDF, the structure of the moment space as well as the canonical moments are presented and the main properties of the two systems of PDEs involving these vectors of moments are analyzed. Moreover, the closure of velocity moments for sprays introduces the pressureless gas formalism, the influence of which is briefly described.

\subsection{Fundamental modeling at the kinetic level}

The dilute spray of droplet is described by a NDF $f(t, \boldsymbol{x}, S, \boldsymbol{v})$, such that $f(t, \boldsymbol{x}, S, \boldsymbol{v}) \mathrm{d} \boldsymbol{x} \mathrm{d} S \mathrm{~d} \boldsymbol{v}$ represents the probable number of droplets located in $\boldsymbol{x}=\left(x_{1}, \ldots, x_{d}\right)$, where $d$ is the dimension of the physical space), with size $S$, and velocity $\boldsymbol{v}^{1}$. As the droplets are assumed to be spherical, we choose the droplet surface to describe the size ${ }^{2}$. The NDF is solution of a Fokker-Planck equation modeling the effects of evaporation, drag, and Brownian motion. From the kinetic level, the phase space of which contains size and velocity variables for aerosols or sprays, we use either a singular perturbation theory based on the Stokes number or a moment method in velocity conditioned on droplet size in order to derive "macroscopic" semi-kinetic system of partial differential equations (PDEs) under the form of a system of conservation laws. For the sake of legibility of the paper, such a derivation is recalled in Appendix 7.A and leads to the following systems of conservation equations.

\footnotetext{
${ }^{1}$ We refer to Doisneau et al [14] and references therein for the treatment of droplet enthalpy.

${ }^{2}$ We could have also chosen to work with their radius, $R$ or their volume, $V$, all corresponding NDF being linked by the relation $f(t, \boldsymbol{x}, R, \boldsymbol{v}) d R=f(t, \boldsymbol{x}, S, \boldsymbol{v}) \mathrm{d} S=f(t, \boldsymbol{x}, V, \boldsymbol{v}) \mathrm{d} V$.
} 


\section{Aerosol}

For aerosols, Brownian motion has to be taken into account. The number density conditioned by droplet size $n(t, \boldsymbol{x}, S)$ satisfies the Smoluchowski equation $[23,6]$ :

$$
\partial_{t} n+\partial_{\boldsymbol{x}}\left(\boldsymbol{u}_{g} n\right)-\partial_{S}(K n)=\partial_{\boldsymbol{x}}\left(\boldsymbol{D} \partial_{\boldsymbol{x}} n\right),
$$

where $\boldsymbol{D}$ is the diffusion operator and its expression is given in Appendix 7.A.

Spray

In the case of spray droplets, because of their Stokes regime $(\mathrm{St} \approx 1)$, the proper droplet dynamics needs to be solved. The gaz/droplet interaction is governed by drag so that the semi-kinetic equation can be written:

$$
\begin{aligned}
\partial_{t} n+\partial_{\boldsymbol{x}}(n \boldsymbol{u})-\partial_{S}(K n) & =0, \\
\partial_{t}(n \boldsymbol{u})+\partial_{\boldsymbol{x}}(n \boldsymbol{u} \otimes \boldsymbol{u}+n \boldsymbol{P})-\partial_{S}(K n \boldsymbol{u})-n \frac{\left(\boldsymbol{u}_{g}-\boldsymbol{u}\right)}{\mathrm{St}} & =0,
\end{aligned}
$$

where $n \boldsymbol{P}=\int(\boldsymbol{v}-\boldsymbol{u}) \otimes(\boldsymbol{v}-\boldsymbol{u}) f \mathrm{~d} v$ is the droplet pressure tensor. A hypothesis on the velocity distribution has to be made in order to obtain a closed equation on the total number density conditioned on size, $n(t, x, S)$. Following the example of what is done in the multi-fluid model $[25,32,8]$, we suppose that there is no dispersion around the mean velocity. In other terms, we "project" $f$ on a distribution with a single velocity conditioned on size: $f(t, \boldsymbol{x}, S, \boldsymbol{v})=n(t, \boldsymbol{x}, S) \delta(\boldsymbol{v}-\boldsymbol{u}(t, \boldsymbol{x}, S))$, leading to $\boldsymbol{P}=0$. This assumption is fully legitimate for small Stokes numbers, as $\boldsymbol{u} \approx \boldsymbol{u}_{g}$ in this case. Nevertheless even if $\mathrm{St} \approx 1$, where physically some velocity slip may occur, this hypothesis leads to very good results $[8,9,27]$. It can be shown $[36,27]$ that other closure assumptions introduced in [11] can be taken into account in order to treat a higher number of velocity moments and thus to treat particle trajectory crossing (PTC) while still describing polydispersity. Such ideas are valid with Eulerian multi-fluid model and lead to good results $[9,27]$. For the sake of presenting the method in the present contribution we rather take the simpler case of a single node quadrature, that leads to pressureless gas dynamics.

\subsection{Size moment equations}

In both cases, moment methods will be used for the simulations. Size moments of order 0 to $N$ are then introduced, thus considering the evolution of the total number density of the droplets, their mean size, their mean size squared (which can be linked to the dispersion in size of the NDF), etc... This moments on the non dimensional interval $[0,1]$ are defined as follows:

$$
m_{k}(t, \boldsymbol{x})=\int_{0}^{1} S^{k} n(t, \boldsymbol{x}, S) \mathrm{d} S .
$$

In the following, we make an additional assumption on the size-velocity correlation. We assume that the velocity of the particles does not depend on size, that is $f(t, \boldsymbol{x}, S, \boldsymbol{v})=n(t, \boldsymbol{x}, S) \delta(\boldsymbol{v}-\boldsymbol{u}(t, \boldsymbol{x}))$. The extension of the present study to size-velocity correlation and cross size/velocity moments is conducted in [47] and requires additional ingredients. Whereas in the Eulerian multi-fluid model, the size-velocity correlation is automatically taken into account, since we have one velocity per section, in the present study we only will have one velocity for the entire size spectrum. However, it will be shown in Section 5, that the description of the size distribution through the use of several moments will allow to have a good description of the spray dynamics in average thanks to an improvement of the global spray relaxation time in the drag force.

Since we aim at describing evaporation and transport only, the diffusion term will be omitted for the present study. Let us notice that this does not involve any loss of generality since a dedicated scheme for diffusion can be further added in the context of an operator splitting algorithm. For the sake of legibility, the evaporation coefficient is assumed to be independent of the droplet size, referring to [37] for more complex evaporation laws. 
From Eq. (1) and system (2), the systems on the moments relative to the EMSM model are written:

$$
\begin{aligned}
& \text { Aerosols } \\
& \left\{\begin{array} { l } 
{ \partial _ { t } ( m _ { 0 } ) + \partial _ { \boldsymbol { x } } ( m _ { 0 } \boldsymbol { u } _ { g } ) = - K n ( S = 0 ) , } \\
{ \partial _ { t } ( m _ { 1 } ) + \partial _ { \boldsymbol { x } } ( m _ { 1 } \boldsymbol { u } _ { g } ) = - K m _ { 0 } , } \\
{ \partial _ { t } ( m _ { 2 } ) + \partial _ { \boldsymbol { x } } ( m _ { 2 } \boldsymbol { u } _ { g } ) = - 2 K m _ { 1 } , } \\
{ \vdots } \\
{ \partial _ { t } ( m _ { N } ) + \partial _ { \boldsymbol { x } } ( m _ { N } \boldsymbol { u } _ { g } ) = - N K m _ { N - 1 } , }
\end{array} \quad \left\{\quad \left\{\begin{array}{rl}
\partial_{t}\left(m_{0}\right)+\partial_{\boldsymbol{x}}\left(m_{0} \boldsymbol{u}\right) & =-K n(S=0), \\
\partial_{t}\left(m_{1}\right)+\partial_{\boldsymbol{x}}\left(m_{1} \boldsymbol{u}\right) & =-K m_{0}, \\
\partial_{t}\left(m_{2}\right)+\partial_{\boldsymbol{x}}\left(m_{2} \boldsymbol{u}\right) & =-2 K m_{1}, \\
& \vdots \\
\partial_{t}\left(m_{N}\right)+\partial_{\boldsymbol{x}}\left(m_{N} \boldsymbol{u}\right) & =-N K m_{N-1}, \\
\partial_{t}\left(m_{1} \boldsymbol{u}\right)+\partial_{\boldsymbol{x}}\left(m_{1} \boldsymbol{u} \otimes \boldsymbol{u}\right)=\boldsymbol{T}-K m_{0} \boldsymbol{u} .
\end{array}\right.\right.\right.
\end{aligned}
$$

The quantity $\boldsymbol{T}$ represents the drag term. It writes $\boldsymbol{T}(t, \boldsymbol{x})=\int_{0}^{1} S n(t, \boldsymbol{x}, S) \frac{\boldsymbol{u}_{g}(t, \boldsymbol{x})-\boldsymbol{u}(t, \boldsymbol{x})}{\mathrm{St}} \mathrm{d} S$ in the Stokes regime considered here (let us however notice that introducing a more complex drag force does not bring any difficulties). The Stokes number being proportional to the droplet surface, let us denote $\theta$ the proportionality coefficient: $\mathrm{St}=\theta S$. The drag force is then written:

$$
\boldsymbol{T}(t, \boldsymbol{x})=\frac{m_{0}}{\theta}\left(\boldsymbol{u}_{g}(t, \boldsymbol{x})-\boldsymbol{u}(t, \boldsymbol{x})\right) .
$$

Let us remark that, if the last equation of (5) had been written on $m_{0} \boldsymbol{u}$, then the drag term would not have been integrable (the NDF $n$ has generally a non zero value at $S=0$ [37]).

Concerning the evaporation term, the resolution of the corresponding system will be detailed in section 4 with an algorithm developed in [37].

Let us then focus on convection of the moments. An algorithm is here designed for the resolution of the following systems:

$$
\begin{gathered}
\text { Aerosols } \\
\left\{\begin{array} { c } 
{ \partial _ { t } ( m _ { 0 } ) + \partial _ { \boldsymbol { x } } ( m _ { 0 } \boldsymbol { u } _ { g } ) = 0 , } \\
{ \partial _ { t } ( m _ { 1 } ) + \partial _ { \boldsymbol { x } } ( m _ { 1 } \boldsymbol { u } _ { g } ) = 0 , } \\
{ \partial _ { t } ( m _ { 2 } ) + \partial _ { \boldsymbol { x } } ( m _ { 2 } \boldsymbol { u } _ { g } ) = 0 , } \\
{ \vdots } \\
{ \partial _ { t } ( m _ { N } ) + \partial _ { \boldsymbol { x } } ( m _ { N } \boldsymbol { u } _ { g } ) = 0 , }
\end{array} \quad \left\{\begin{array}{cc}
\partial_{t}\left(m_{0}\right)+\partial_{\boldsymbol{x}}\left(m_{0} \boldsymbol{u}\right) & =0 \\
\partial_{t}\left(m_{1}\right)+\partial_{\boldsymbol{x}}\left(m_{1} \boldsymbol{u}\right) & =0 \\
\partial_{t}\left(m_{2}\right)+\partial_{\boldsymbol{x}}\left(m_{2} \boldsymbol{u}\right) & =0 \\
& \vdots \\
\partial_{t}\left(m_{N}\right)+\partial_{\boldsymbol{x}}\left(m_{N} \boldsymbol{u}\right) & =0 \\
\partial_{t}\left(m_{1} \boldsymbol{u}\right)+\partial_{\boldsymbol{x}}\left(m_{1} \boldsymbol{u} \otimes \boldsymbol{u}\right) & =0
\end{array}\right.\right.
\end{gathered}
$$

However, before designing such algorithm, some key properties of the moment space and of the systems of PDEs have to be recalled. This is the subject of the next two sections.

\subsection{Moment space and canonical moments}

The major issue of the numerical scheme developed here is to keep the integrity of the moment sequence $\left(m_{0}, \ldots, m_{N}\right)^{t}$. Indeed, Wright [50] showed that independent transport of moments with algorithms of order greater than one in space, can result in the generation of invalid moment sets.

So, let us first define the moment space. If $\mathcal{P}$ denotes the set of all probability measures on the Borel sets of the interval $[0,1]$, then the $N$ th-moment space $\widetilde{\mathcal{M}}_{N}$ on the interval $[0,1]$ denotes the following set of moment vector of dimension $N, \widetilde{\mathcal{M}}_{N} \subset[0,1]^{N}$ :

$$
\widetilde{\mathcal{M}}_{N}=\left\{\boldsymbol{c}_{n}(\mu) \mid \mu \in \mathcal{P}\right\}, \quad \mathbf{c}_{N}(\mu)=\left(c_{1}(\mu), \ldots, c_{N}(\mu)\right)^{t}, \quad c_{k}(\mu)=\int_{0}^{1} x^{k} d \mu(x) .
$$

Since we consider probability measures, we always have $c_{0}=1$. In our case we are dealing with a number density function, thus $m_{0}$ is the droplet number density. One can associate to the moment vector $\mathcal{M}=\left(m_{0}, m_{1}, \ldots, m_{N}\right)^{t} \in \mathbf{R}^{N+1}$ the normalized moment vector $\boldsymbol{c}_{n}=\left(c_{1}, \ldots, c_{n}\right)^{t}$ such as $c_{k}=\frac{m_{k}}{m_{0}}$. 
Let us then denote $\mathcal{M}_{N}$ the $N$ th-moment space of such moment vectors $\mathcal{M}$. Such a space is convex but has a rather complex geometry. In order to study some of its useful properties, we will take benefit from some derived quantities : the Hankel determinants and the canonical moments [12].

The Hankel determinants are defined by:

$$
\underline{H}_{2 m+d}=\left|\begin{array}{ccc}
c_{d} & \ldots & c_{m+d} \\
\vdots & & \vdots \\
c_{m+d} & \ldots & c_{2 m+d}
\end{array}\right|, \quad \bar{H}_{2 m+d}=\left|\begin{array}{ccc}
c_{1-d}-c_{2-d} & \ldots & c_{m}-c_{m+1} \\
\vdots & \vdots & \vdots \\
c_{m}-c_{m+1} & \ldots & c_{2 m-1+d}-c_{2 m+d}
\end{array}\right|,
$$

with $d=0,1 ; m \geq 0, \underline{H}_{-1}=\bar{H}_{-1}=\underline{H}_{0}=\bar{H}_{0}=1$. Necessary and sufficient conditions for a moment vector $\mathcal{M}=\left(m_{0}, m_{1}, \ldots, m_{N}\right)$ to be in the moment space $\mathcal{M}_{N}$ (i.e. for the existence of a necessarily non unique NDF $n(t, \boldsymbol{x}, S))$ are non negative Hankel determinants $\underline{H}_{i}$ and $\bar{H}_{i}$ [12]. Moreover, at least one of the Hankel determinant is zero for a moment vector lying on the border of the moment space. Checking if a $N+1$ component vector belongs to the moment space is quite tedious when $N \geq 2$. However, the Hankel determinants provide algebraic relations to quickly determine if a vector belongs to the moment space. Moreover they enable to derive quantities called canonical moments, linked with a one-to-one mapping to the moment space. The canonical moment space has the very convenient property of since it maps exactly the cube $] 0,1\left[^{k}\right.$, and not in a strict subset of it such as the moment space. Therefore, in order to check if the moment space is preserved, it is more practical to work with the canonical moments.

Let us introduce the set $P\left(\boldsymbol{c}_{k-1}\right)$ of all probability measures on $[0,1]$ whose moments of order up to $k-1$ are $c_{j}$. Dette and Studden [12] have given the definition of the canonical moments of order $k$ $(k \geq 1)$ :

$$
p_{k}=\frac{c_{k}-c_{k}^{-}\left(\boldsymbol{c}_{k-1}\right)}{c_{k}^{+}\left(\boldsymbol{c}_{k-1}\right)-c_{k}^{-}\left(\boldsymbol{c}_{k-1}\right)},
$$

with

$$
c_{k}^{+}\left(\boldsymbol{c}_{k-1}\right)=\max _{\mu \in P\left(\boldsymbol{c}_{k-1}\right)} c_{k}(\mu), \quad c_{k}^{-}\left(\boldsymbol{c}_{k-1}\right)=\min _{\mu \in P\left(\boldsymbol{c}_{k-1}\right)} c_{k}(\mu)
$$

$c_{k}(\mu)$ being the moment of order $k$ of the probability measure $\mu$. The quantities $c_{k}^{+}\left(\boldsymbol{c}_{k-1}\right)$ and $c_{k}^{-}\left(\boldsymbol{c}_{k-1}\right)$ are respectively the upper and lower boundary of the admissible interval for the moment $c_{k}$ of order $k$, the lower order moments being known. If $c_{k}(\mu)=c_{k}^{+}\left(\boldsymbol{c}_{k-1}\right)$ or $c_{k}(\mu)=c_{k}^{-}\left(\boldsymbol{c}_{k-1}\right)$ for $\mu \in P\left(\boldsymbol{c}_{k-1}\right)$, or equivalently if $p_{k}=0$ or $p_{k}=1$, the measure $\mu$ is a sum of Dirac distributions and the vector $\boldsymbol{c}_{k}$ belongs to the boundary of the moment space.

The $c_{k}^{+}-c_{k}^{-}$and canonical moments are expressed from the Hankel determinants in [12], for $k \geq 1$ :

$$
c_{k}^{+}-c_{k}^{-}=\frac{\underline{H}_{k-1} \bar{H}_{k-1}}{\underline{H}_{k-2} \bar{H}_{k-2}}, \quad p_{k}=\frac{\underline{H}_{k} \bar{H}_{k-2}}{\underline{H}_{k-1} \bar{H}_{k-1}} .
$$

But practically, the canonical moments are recursively determined from the lower order ones. On the size interval $[0,1]$, The expressions of the first three canonical moments are:

$$
p_{1}=c_{1}, \quad p_{2}=\frac{c_{2}-c_{1}^{2}}{c_{1}\left(1-c_{1}\right)}, \quad p_{3}=\frac{\left(1-c_{1}\right)\left(c_{1} c_{3}-c_{2}^{2}\right)}{\left(c_{2}-c_{1}^{2}\right)\left(c_{1}-c_{2}\right)} .
$$

The canonical moments have two major properties which make them attractive to work with. First, according to the definition (9), each canonical moment independently lies in the interval ]0,1[ for a moment vector in the interior of the moment space. It is thus straightforward to figure out if the associated moment vector belongs to the moment space. Secondly, the canonical moments remain invariant under linear transformation of the distribution, i.e for all $k \geq 1, p_{k}(f)=p_{k}\left(f_{S_{\min } S_{\max }}\right)$, where $f_{S_{\min } S_{\max }}$ denotes the distribution induced by the linear transformation $S=S_{\min }+\left(S_{\max }-S_{\min }\right) x$ of $[0,1]$ onto $\left[S_{\min }, S_{\max }\right]$ (we refer to [12] for the proof). That is the reason why we can work on the size interval $[0,1]$ without loss of generality.

In addition to the two previous properties, another property given from systems (7) will be very useful when designing the numerical scheme. 
Proposition 1. Let $\boldsymbol{u}(t, \boldsymbol{x})$ be a $C^{1}$ function. If the moment vector $\left(m_{0}, m_{1}, \ldots, m_{N}\right)$ is a $C^{1}$ function of $t$ and $\boldsymbol{x}$ belonging to the interior of the moment space and such that

$$
\left\{\begin{array}{c}
\partial_{t}\left(m_{0}\right)+\partial_{\boldsymbol{x}}\left(m_{0} \boldsymbol{u}\right)=0 \\
\partial_{t}\left(m_{1}\right)+\partial_{\boldsymbol{x}}\left(m_{1} \boldsymbol{u}\right)=0 \\
\partial_{t}\left(m_{2}\right)+\partial_{\boldsymbol{x}}\left(m_{2} \boldsymbol{u}\right)=0 \\
\vdots \\
\partial_{t}\left(m_{N}\right)+\partial_{\boldsymbol{x}}\left(m_{N} \boldsymbol{u}\right)=0
\end{array}\right.
$$

then the corresponding canonical moments are transported quantities, which means that they verify the transport equation: $\partial_{t} p_{k}+\boldsymbol{u} \partial_{\boldsymbol{x}} p_{k}=0$.

Proof . - According to Eq. (11), $p_{k}$ is a rational fraction of the moments $c_{k}$. Moreover, since the moment vector is in the interior of the moment space, the denominator can never be equal to zero. Therefore, $p_{k}\left(c_{k}\right)$ is differentiable relative to each $c_{j, j \leq k}$. Thus we have the relation:

$$
\partial_{t} p_{k}+\boldsymbol{u} \partial_{x} p_{k}=\sum_{j \leq k} \partial_{c_{j}} p_{k}\left[\partial_{t} c_{j}+\boldsymbol{u} \partial_{x} c_{j}\right]
$$

But since the $c_{j}$ are transported quantities, we have $\partial_{t} p_{k}+\boldsymbol{u} \partial_{x} p_{k}=0$.

\subsection{Pressureless gas dynamics}

Let us remark that the system (7) for the spray contains the pressureless gas dynamics system which has been studied by several authors $[2,4]$. Indeed, this system can be written:

$$
\partial_{t} U+\partial_{\boldsymbol{x}} F(U)=0, \quad \mathbf{A}(U)=\partial_{U} F(U),
$$

where

$$
U=\left(m_{0}, \ldots, m_{N}, m_{1} u\right)^{t}, \quad F=\left(m_{0} u, \ldots, m_{N} u, m_{1} u^{2}\right)^{t} .
$$

In the pressureless gas system, the Jacobian matrix of the flux $\mathbf{A}$ is a Jordan block, the system is weakly hyperbolic. A main feature of this weakly hyperbolic system is the development of singularities for the conserved quantities called $\delta$-shocks, and the emergence of the vacuum state, even when the initial distribution is regular. Such a system can be seen as a particular case of a more general class of systems called quadrature-based moment methods in velocity space (see [5] and references therein). Besides, as mentioned before, such quadrature-based high order moment methods allow to deal with PTC as well as polydispersity as long as the assumption of size-velocity de-correlation is justified as done in [36, 26] with the present high order moment method in size. In the following we will only consider pressureless gas dynamics since the coupling with high order moment in velocity is straightforward.

The key issue is thus to design a robust numerical scheme with second order in time and space for the advection of the moments satisfying the realizability condition in both the case of aerosols as well as in the case of sprays where singularity formation can occur. As in many cases, the construction of the discretization will rely on specific properties of the continuous system of partial differential equations we have derived so far. The following section exposes the characteristics of the scheme preserving the moment space, and capturing the singularities introduced by the pressureless gas dynamics.

\section{A new finite volume kinetic numerical scheme preserving the moment space}

In this section, we present the numerical scheme used to discretize system (7) in the case of an aerosol, and its extension to treat the case of a spray. Because of the conservative form of system (7), the finite-volume method [33] is a natural candidate for its discretization. Moreover, since the computations we present are enforced in a cartesian mesh, we use a dimensional splitting algorithm explained by Strang [45], preserving the second order in time of the scheme and also its mathematical properties. An alternated Lie splitting can also be used with the advantage of using the 1D transport algorithm with the same time step in each direction $[8,33]$, while still preserving the second order in time. So, without 
loss of generality, the scheme is then presented in a one dimensional framework, with the addition of the transport equation of a passive scalar corresponding to an eventual velocity component in another direction. In the following we will fully take advantage of the fact that we work with structured meshes in order to build a realizable second order in time and space advection scheme with minimal numerical diffusion, which is strictly stemming from the reconstruction step in the finite volume cells. Let us notice however, that the ingredients we introduce in order to obtain such a scheme can be used in order to design an extension of the scheme to unstructured meshes. Since this is not the primary scope of the paper, we present the principles of such an extension in Appendix 7.C.

Usually, high order cell center finite volume methods use some non-constant reconstructions of the variables to evaluate the fluxes at the interfaces of the cells. Two difficulties have to be overcome. The first one concerns the spatial reconstruction of the moments in order to keep the integrity of the moment set. A second difficulty concerns the temporal integration scheme in order to perform computation at $C F L=1$. Using basic explicit time intergrators with Runge-Kutta types schemes in order to increase the order in time often requires the CFL number be smaller than one.

In order to overcome this restriction, we design a kinetic-based numerical scheme using the ideas developed by Bouchut [3]. The time solver is based on an exact resolution of the PDE in time which relies on a micro-macro equivalence property. An exact computation of the fluxes is obtained using the fact that the equations on moments can be derived from the kinetic equivalent equation and thus leads to a second order in time and space with minimal numerical diffusion.

\subsection{General form of the kinetic schemes}

In a similar manner as in [3], a kinetic scheme is developed, based on the equivalence between the "macroscopic" system and a kinetic equation. The "macroscopic" equation on the size distribution $n(t, x, S)$ is:

$$
\partial_{t} n+\partial_{x}(n u)=0
$$

where $u$ is the gas velocity for aerosols and the droplet velocity for the spray in the direction we are dealing with. A passive scalar $v$ can also be considered, representing an eventual other direction in the framework of dimensional splitting for a $2 \mathrm{D}$ or $3 \mathrm{D}$ computation. Both these variables are then a solution of:

$$
\begin{aligned}
& \partial_{t}(n u)+\partial_{x}\left(n u^{2}\right)=0, \\
& \partial_{t}(n v)+\partial_{x}(n u v)=0 .
\end{aligned}
$$

This "macroscopic" system is equivalent to the kinetic equation on $f(t, x, \xi, \zeta, S)$ :

$$
\partial_{t} f+\partial_{x}(\xi f)=0
$$

with $f(t, x, \xi, \zeta, S)=n(t, x, S) \delta(\xi-u(t, x)) \delta(\zeta-v(t, x))$. This kinetic equation has the exact solution $f(t, x, \xi, \zeta, S)=f(0, x-\xi t, \xi, \zeta, S)$. The strategy to develop the scheme is then the same one as in [3], with the difference that here, the "macroscopic" system of interest $(7)$ is not directly $(17)$ or $(17,18)$ but a system induced by taking the size moments of these equations.

In order to obtain discrete values over a mesh of constant size $\Delta x$, one defines the averages $m_{k, j}^{n}, u_{j}^{n}$ and $v_{j}^{n}$ for inertial droplets, with the usual definitions:

$$
\begin{aligned}
m_{k, j}^{n} & =\frac{1}{\Delta x} \int_{x_{j-1 / 2}}^{x_{j+1 / 2}} m_{k}\left(t_{n}, x\right) \mathrm{d} x, \quad k=0, \ldots, N, \\
\boldsymbol{q}_{j}^{n} & =m_{1, j}^{n}\left(\begin{array}{c}
u_{j}^{n} \\
v_{j}^{n}
\end{array}\right)=\frac{1}{\Delta x} \int_{x_{j-1 / 2}}^{x_{j+1 / 2}} m_{1}\left(t_{n}, x\right)\left(\begin{array}{c}
u\left(t_{n}, x\right) \\
v\left(t_{n}, x\right)
\end{array}\right) \mathrm{d} x,
\end{aligned}
$$

Let us denote $\mathcal{M}_{N}=\left(m_{0}, \ldots, m_{N}\right)^{t}$ the vector of moments. The discretized equations are obtained in a conservative form by integrating Eq. (20) multiplied by $\left(1, S, \ldots, S^{N}, \xi\right)^{t}$ over $(t, x, \xi, S) \in\left(t_{n}, t_{n+1}\right) \times$ $\left(x_{j-1 / 2}, x_{j+1 / 2}\right) \times \mathbb{R} \times(0,1):$

$$
\begin{array}{r}
\mathcal{M}_{N, j}^{n+1}=\mathcal{M}_{N, j}^{n}-\frac{\Delta t}{\Delta x}\left(\boldsymbol{F}_{j+1 / 2}-\boldsymbol{F}_{j-1 / 2}\right), \\
\boldsymbol{q}_{j}^{n+1}=\boldsymbol{q}_{j}^{n}-\frac{\Delta t}{\Delta x}\left(\boldsymbol{G}_{j+1 / 2}-\boldsymbol{G}_{j-1 / 2}\right),
\end{array}
$$


where the fluxes for the size moments $\boldsymbol{F}_{j+1 / 2}$ can be decomposed in $\boldsymbol{F}_{j+1 / 2}=\boldsymbol{F}_{j+1 / 2}^{+}+\boldsymbol{F}_{j+1 / 2}^{-}$with:

$$
\boldsymbol{F}_{j+1 / 2}^{ \pm}=\frac{1}{\Delta t} \int_{t_{n}}^{t_{n+1}} \int_{ \pm \xi \geq 0} \int_{0}^{1} \int_{\mathbf{R}}\left(\begin{array}{c}
1 \\
S \\
\vdots \\
S^{N}
\end{array}\right) \xi f\left(t, x_{j+1 / 2}, \xi, \zeta, S\right) \mathrm{d} \zeta \mathrm{d} S \mathrm{~d} \xi \mathrm{d} t
$$

and in the same way, the flux for the momentum in the case of inertial droplets is $\boldsymbol{G}_{j+1 / 2}=\boldsymbol{G}_{j+1 / 2}^{+}+$ $\boldsymbol{G}_{j+1 / 2}^{-}$with:

$$
\boldsymbol{G}_{j+1 / 2}^{ \pm}=\frac{1}{\Delta t} \int_{t_{n}}^{t_{n+1}} \int_{ \pm \xi \geq 0} \int_{0}^{1} \int_{\mathbf{R}} S\left(\begin{array}{c}
\xi \\
\zeta
\end{array}\right) \xi f\left(t, x_{j+1 / 2}, \xi, \zeta, S\right) \mathrm{d} \zeta \mathrm{d} S \mathrm{~d} \xi \mathrm{d} t
$$

To evaluate the fluxes, the exact solution of the kinetic scheme is used:

$$
\begin{aligned}
\left(\begin{array}{c}
\boldsymbol{F}_{j+1 / 2}^{ \pm} \\
\boldsymbol{G}_{j+1 / 2}^{ \pm}
\end{array}\right) & =\frac{1}{\Delta t} \int_{0}^{\Delta t} \int_{ \pm \xi \geq 0} \int_{0}^{1}\left(\begin{array}{c}
1 \\
S \\
\vdots \\
S^{N} \\
S \xi \\
S v\left(t_{n}, x_{j+1 / 2}-\xi t\right)
\end{array}\right) \xi n\left(t_{n}, x_{j+1 / 2}-\xi t, S\right) \delta\left(\xi-u\left(t_{n}, x_{j+1 / 2}-\xi t\right)\right) \mathrm{d} S \mathrm{~d} \xi \mathrm{d} t \\
= & \frac{1}{\Delta t} \int_{0}^{\Delta t} \int_{ \pm \xi \geq 0}\left(\begin{array}{c}
m_{0}\left(t_{n}, x_{j+1 / 2}-\xi t\right) \\
m_{1}\left(t_{n}, x_{j+1 / 2}-\xi t\right) \\
\vdots \\
m_{N}\left(t_{n}, x_{j+1 / 2}-\xi t\right) \\
m_{1}\left(t_{n}, x_{j+1 / 2}-\xi t\right) \xi \\
\left(m_{1} v\right)\left(t_{n}, x_{j+1 / 2}-\xi t\right)
\end{array}\right) \xi \delta\left(\xi-u\left(t_{n}, x_{j+1 / 2}-\xi t\right)\right) \mathrm{d} \xi \mathrm{d} t
\end{aligned}
$$

Let us develop the computation for $\left(\boldsymbol{F}_{j+1 / 2}^{+}, \boldsymbol{G}_{j+1 / 2}^{+}\right)$. A change of variable between $t$ and $x=x_{j+1 / 2}-\xi t$ gives:

$$
\begin{aligned}
\left(\begin{array}{c}
\boldsymbol{F}_{j+1 / 2}^{+} \\
\boldsymbol{G}_{j+1 / 2}^{+}
\end{array}\right) & =\frac{1}{\Delta t} \int_{\xi \geq 0} \int_{x_{j+1 / 2}-\xi \Delta t}^{x_{j+1 / 2}}\left(\begin{array}{c}
m_{0}\left(t_{n}, x\right) \\
m_{1}\left(t_{n}, x\right) \\
\vdots \\
m_{N}\left(t_{n}, x\right) \\
m_{1}\left(t_{n}, x\right) \xi \\
\left(m_{1} v\right)\left(t_{n}, x\right)
\end{array}\right) \delta\left(\xi-u\left(t_{n}, x\right)\right) \mathrm{d} \xi \mathrm{d} x \\
& =\frac{1}{\Delta t} \int_{x_{j-1 / 2}}^{x_{j+1 / 2}}\left(\begin{array}{c}
m_{0}\left(t_{n}, x\right) \\
m_{1}\left(t_{n}, x\right) \\
\vdots \\
m_{N}\left(t_{n}, x\right) \\
m_{1}\left(t_{n}, x\right) u\left(t_{n}, x\right) \\
m_{1}\left(t_{n}, x\right) v\left(t_{n}, x\right)
\end{array}\right) \mathbb{I}_{\left\{x, x_{j+1 / 2}-u\left(t_{n}, x\right) \Delta t \leq x \leq x_{j+1 / 2}\right\}}(x) \mathrm{d} x,
\end{aligned}
$$

the last expression being valid under the CFL condition: $\Delta t \sup _{x}\left|u\left(t_{n}, x\right)\right| \leq \Delta x$. In the same way, the 
other part of the fluxes are:

$$
\left(\begin{array}{c}
\boldsymbol{F}_{j+1 / 2}^{-} \\
\boldsymbol{G}_{j+1 / 2}^{-}
\end{array}\right)=-\frac{1}{\Delta t} \int_{x_{j+1 / 2}}^{x_{j+3 / 2}}\left(\begin{array}{c}
m_{0}\left(t_{n}, x\right) \\
m_{1}\left(t_{n}, x\right) \\
\vdots \\
m_{N}\left(t_{n}, x\right) \\
m_{1}\left(t_{n}, x\right) u\left(t_{n}, x\right) \\
m_{1}\left(t_{n}, x\right) v\left(t_{n}, x\right)
\end{array}\right) \mathbb{I}_{\left\{x, x_{j+1 / 2} \leq x \leq x_{j+1 / 2}-u\left(t_{n}, x\right) \Delta t\right\}}(x) \mathrm{d} x .
$$

The fluxes are then written with the variables of interest: the moments $m_{k}$ of the size distribution $n$. The difficulty now is to reconstruct the moments $m_{k}\left(t_{n},.\right)$ from the $m_{k, j}^{n}$ in such a way that the moment space is preserved. In order to show how this scheme is built in a simple case, we will first compute the convective fluxes in the context of a first order kinetic scheme.

\subsection{First order kinetic scheme}

For a first order kinetic scheme, piecewise constant data are reconstructed:

$$
\text { for } x_{j-1 / 2}<x<x_{j+1 / 2} \quad\left\{\begin{aligned}
m_{k}\left(t_{n}, x\right) & =m_{k, j}^{n}, \quad k=0, \ldots, N \\
u\left(t_{n}, x\right) & =u_{j}^{n} \\
v\left(t_{n}, x\right) & =v_{j}^{n}
\end{aligned}\right.
$$

Under the CFL condition $\Delta t \sup _{j}\left|u_{j}^{n}\right| \leq \Delta x$, the expression of the fluxes $(26,27)$ are straightforward:

$$
\boldsymbol{F}_{j+1 / 2}^{+}=\mathcal{M}_{N, j}^{n}\left(u_{j}^{n}\right)_{+}, \quad \boldsymbol{G}_{j+1 / 2}^{+}=m_{1, j}^{n}\left(\begin{array}{c}
u_{j}^{n} \\
v_{j}^{n}
\end{array}\right)\left(u_{j}^{n}\right)_{+},
$$

and

$$
\boldsymbol{F}_{j+1 / 2}^{-}=\mathcal{M}_{N, j+1}^{n}\left(u_{j+1}^{n}\right)_{-}, \quad \boldsymbol{G}_{j+1 / 2}^{-}=m_{1, j+1}^{n}\left(\begin{array}{c}
u_{j+1}^{n} \\
v_{j+1}^{n}
\end{array}\right)\left(u_{j+1}^{n}\right)_{-},
$$

with the convention $u_{+}=\max \{u, 0\}, u_{-}=\min \{u, 0\}$.

With piecewise constant reconstructions, the first order scheme considerably simplifies the reconstruction step as it eludes the problem of preservation of the moment space which arises when non constant reconstruction are considered. Indeed, this first order scheme can be written:

$$
\begin{aligned}
\mathcal{M}_{N, j}^{n+1} & =\left(1-\frac{\Delta t}{\Delta x}\left|u_{j}^{n}\right|\right) \mathcal{M}_{N, j}^{n}-\frac{\Delta t}{\Delta x}\left(u_{j+1}^{n}\right)_{-} \mathcal{M}_{N, j+1}^{n}+\frac{\Delta t}{\Delta x}\left(u_{j-1}^{n}\right)_{+} \mathcal{M}_{N, j-1}^{n} \\
& =\int_{0}^{1}\left(\begin{array}{c}
1 \\
S \\
\vdots \\
S^{N}
\end{array}\right)\left[\left(1-\frac{\Delta t}{\Delta x}\left|u_{j}^{n}\right|\right) f_{j}-\frac{\Delta t}{\Delta x}\left(u_{j+1}^{n}\right)_{-} f_{j+1}+\frac{\Delta t}{\Delta x}\left(u_{j-1}^{n}\right)_{+} f_{j-1}\right](S) \mathrm{d} S,
\end{aligned}
$$

where $f_{j}$ is a distribution function with the moments of order 0 to $N$ given by $\mathcal{M}_{N, j}^{n}$. Under the CFL condition, the coefficients before each $f_{j}$ are non negative and then, $\mathcal{M}_{N, j}^{n+1}$ is a moment vector. In the case of spray, the first order scheme also satisfies the maximum principle on the velocity. Other properties, like entropy inequalities or the TVD property on the velocity, can also be proved, see [2].

The first order scheme is easy to design, and robust, but, as it is well known, this scheme brings in a lot of numerical diffusion. We thus aim at designing a second order in time and space kinetic scheme with exact time integration of the fluxes at the inferfaces.

\subsection{Second order kinetic scheme}

For the purpose of a second order scheme, piecewise linear reconstructions are considered. If it can be done with the number density $m_{0}$ and the velocity $u$ as in [3], normalized moments cannot be independently reconstructed [50]. However, reconstructing one by one the canonical moments enables 
to preserve the integrity of the moment set at each point of the cell. Indeed, canonical moments are proven to be transported quantities by system (7), they satisfy a maximum principle. They also have the property, as explained in (2.3), to live in the full set $] 0,1{ }^{k}$. In the context of this paper, we will restrict ourselves on the case $N=3$. A greater value for $N$ could be considered without difficulty but would lead to more complex algebra and computational cost without a dramatic improvement of the accuracy of the method.

\subsubsection{Reconstruction}

The reconstruction writes:

$$
\text { for } x_{j-1 / 2}<x<x_{j+1 / 2} \quad\left\{\begin{aligned}
m_{0}(x)=m_{0, j}^{n}+D_{m_{0, j}}\left(x-x_{j},\right) \\
p_{1}(x)=\overline{p_{1, j}}+D_{p_{1, j}}\left(x-x_{j}\right), \\
p_{2}(x)=\overline{p_{2, j}}+D_{p_{2, j}}\left(x-x_{j}\right), \\
p_{3}(x)=\overline{p_{3, j}}+D_{p_{3, j}}\left(x-x_{j}\right), \\
u(x)=\overline{u_{j}}+D_{u_{j}}\left(x-x_{j}\right), \\
v(x)=\overline{v_{j}}+D_{v_{j}}\left(x-x_{j}\right),
\end{aligned}\right.
$$

where $x_{j}=\left(x_{j+1 / 2}+x_{j-1 / 2}\right) / 2$ is the center of the $j^{\text {th }}$ cell and where, to simplify the notation, the $t_{n}$ dependance of each function is implicit. The quantities with bars are different from the canonical moments $p_{i, j}$ corresponding to the moment vector $\mathcal{M}_{3, j}^{n}$ and from the mean velocities $u_{j}^{n}$ and $v_{j}^{n}$. In order to have the conservation property, they are defined in such a way that (according to (12)):

$$
\begin{aligned}
& m_{1, j}^{n}=\frac{1}{\Delta x} \int_{x_{j-1 / 2}}^{x_{j+1 / 2}} m_{0}(x) p_{1}(x) \mathrm{d} x \\
& m_{2, j}^{n}=\frac{1}{\Delta x} \int_{x_{j-1 / 2}}^{x_{j+1 / 2}} m_{0}(x) p_{1}(x)\left[\left(1-p_{1}\right) p_{2}+p_{1}\right](x) \mathrm{d} x \\
& m_{3, j}^{n}=\frac{1}{\Delta x} \int_{x_{j-1 / 2}}^{x_{j+1 / 2}} m_{0}(x) p_{1}(x)\left\{\left(1-p_{1}\right)\left(1-p_{2}\right) p_{2} p_{3}+\left[\left(1-p_{1}\right) p_{2}+p_{1}\right]^{2}\right\}(x) \mathrm{d} x
\end{aligned}
$$

$$
\begin{aligned}
& m_{1, j}^{n} u_{j}^{n}=\frac{1}{\Delta x} \int_{x_{j-1 / 2}}^{x_{j+1 / 2}} m_{0}(x) p_{1}(x) u(x) \mathrm{d} x \\
& m_{1, j}^{n} v_{j}^{n}=\frac{1}{\Delta x} \int_{x_{j-1 / 2}}^{x_{j+1 / 2}} m_{0}(x) p_{1}(x) v(x) \mathrm{d} x .
\end{aligned}
$$

For the first canonical moment, it is easy to see that:

$$
\overline{p_{1, j}}=\frac{m_{1, j}^{n}}{m_{0, j}^{n}}-\frac{D_{p_{1, j}} D_{m_{0, j}}}{m_{0, j}^{n}} \frac{\Delta x^{2}}{12}
$$

When considering higher order canonical moments $\overline{p_{2}}$ and $\overline{p_{3}}$ and the velocity $\overline{u_{j}}$, their expression are more difficult to figure out, since high order polynomials must be integrated, (up to order 4 for $\overline{p_{2}}$ and $\overline{u_{j}}$, and 6 for $\left.\overline{p_{3}}\right)$. Their expression are written:

$$
\overline{u_{j}}=u_{j}^{n}+b_{u, j} D_{u_{j}}, \quad \overline{v_{j}}=v_{j}^{n}+b_{v, j} D_{v_{j}}, \quad \overline{p_{2, j}}=a_{2, j}+b_{2, j} D_{p_{2, j}}, \quad \overline{p_{3, j}}=a_{3, j}+b_{3, j} D_{p_{3, j}},
$$

where $b_{u, j}=b_{v, j}$ is independent of $D_{u_{j}}$ and $D_{v_{j}}, a_{2, j}$ and $b_{2, j}$ are independent of $D_{p_{2, j}}$ and $a_{3, j}$ and $b_{3, j}$ are independent of $D_{p_{3, j}}$. They are given by:

$$
b_{u, j} \int_{x_{j-1 / 2}}^{x_{j+1 / 2}} m_{0}(x) p_{1}(x) \mathrm{d} x=-\int_{x_{j-1 / 2}}^{x_{j+1 / 2}} m_{0}(x) p_{1}(x)\left(x-x_{j}\right) \mathrm{d} x,
$$




$$
\begin{aligned}
a_{2, j} \int_{x_{j-1 / 2}}^{x_{j+1 / 2}}\left\{m_{0} p_{1}\left[1-p_{1}\right]\right\}(x) \mathrm{d} x & =m_{2, j}^{n} \Delta x-\int_{x_{j-1 / 2}}^{x_{j+1 / 2}} m_{0}(x) p_{1}^{2}(x) \mathrm{d} x, \\
b_{2, j} \int_{x_{j-1 / 2}}^{x_{j+1 / 2}}\left\{m_{0} p_{1}\left[1-p_{1}\right]\right\}(x) \mathrm{d} x & =-\int_{x_{j-1 / 2}}^{x_{j+1 / 2}}\left\{m_{0} p_{1}\left[1-p_{1}\right]\right\}(x)\left(x-x_{j}\right) \mathrm{d} x,
\end{aligned}
$$

and

$$
\begin{aligned}
& a_{3, j} \int_{x_{j-1 / 2}}^{x_{j+1 / 2}}\left\{m_{0} p_{1}\left(1-p_{1}\right)\left(1-p_{2}\right) p_{2}\right\}(x) \mathrm{d} x=m_{3, j}^{n} \Delta x-\int_{x_{j-1 / 2}}^{x_{j+1 / 2}}\left\{m_{0} p_{1}\left[\left(1-p_{1}\right) p_{2}+p_{1}\right]^{2}\right\}(x) \mathrm{d} x \\
& b_{3, j} \int_{x_{j-1 / 2}}^{x_{j+1 / 2}}\left\{m_{0} p_{1}\left(1-p_{1}\right)\left(1-p_{2}\right) p_{2}\right\}(x) \mathrm{d} x=-\int_{x_{j-1 / 2}}^{x_{j+1 / 2}}\left\{m_{0} p_{1}\left(1-p_{1}\right)\left(1-p_{2}\right) p_{2}\right\}(x)\left(x-x_{j}\right) \mathrm{d} x .
\end{aligned}
$$

The calculation of these coefficients is achieved using Maple (Maplesoft, a division of Waterloo Maple, Inc 2007) and are directly exported as Fortran 90 subroutines. Their expression is quite heavy, but, as it is just an algebraic relation, the corresponding CPU cost is low. In a general way, let us write $\overline{p_{i, j}}=a_{i, j}+b_{i, j} D_{p_{i, j}}$ for $i=1,2,3$.

\subsubsection{Slope limitation}

Once the conservativity of the scheme is ensured, the slopes are determined using limiters in order to satisfy maximum principles for the transported quantities and positivity for the number density. First, for the positivity of number density, the slope $D_{m_{0, j}}$ must verify;

$$
\left|D_{m_{0, j}} \frac{\Delta x}{2}\right|<m_{0, j}^{n}
$$

To guarantee the maximum principle on the canonical moments $(i=1,2,3)$ :

$$
r_{i j} \leq p_{i}(x) \leq R_{i j}, \quad x \in\left(x_{j-1 / 2}, x_{j+1 / 2}\right),
$$

where $r_{i j}=\min \left(p_{i, j-1}, p_{i, j}, p_{i, j+1}\right)$ and $R_{i j}=\max \left(p_{i, j-1}, p_{i, j}, p_{i, j+1}\right)$, we must have:

$$
\left\{\begin{array}{l}
r_{i, j} \leq a_{i, j}+b_{i, j} D_{p_{i, j}}+\frac{\Delta x}{2} D_{p_{i, j}} \leq R_{i, j}, \\
r_{i, j} \leq a_{i, j}+b_{i, j} D_{p_{i, j}}-\frac{\Delta x}{2} D_{p_{i, j}} \leq R_{i, j} .
\end{array}\right.
$$

From (35), (39) and (41), it is easy to see that $\left|b_{i, j}\right|<\Delta x / 2$ for all $i=1,2,3$. The slopes must then verify:

$$
\left\{\begin{array}{l}
D_{p_{i, j}} \leq \min \left(\frac{R_{i, j}-a_{i, j}}{b_{i, j}+\Delta x / 2}, \frac{a_{i, j}-r_{i, j}}{\Delta x / 2-b_{i, j}}\right), \\
D_{p_{i, j}} \geq \min \left(\frac{r_{i, j}-a_{i, j}}{b_{i, j}+\Delta x / 2}, \frac{a_{i, j}-R_{i, j}}{\Delta x / 2-b_{i, j}}\right) .
\end{array}\right.
$$

In practice, we use the following slope limiter to satisfy all the conditions:

$$
\begin{aligned}
D_{m_{0, j}} & =\frac{1}{2}\left(\operatorname{sgn}\left(m_{0, j+1}^{n}-m_{0, j}^{n}\right)+\operatorname{sgn}\left(m_{0, j}^{n}-m_{0, j-1}^{n}\right)\right) \times \min \left(\frac{\left|m_{0, j+1}^{n}-m_{0, j}^{n}\right|}{\Delta x}, \frac{\left|m_{0, j}^{n}-m_{0, j-1}^{n}\right|}{\Delta x}, \frac{2 m_{0, j}^{n}}{\Delta x}\right) \\
D_{p_{i, j}} & =\frac{1}{2}\left(\operatorname{sgn}\left(p_{i, j+1}-p_{i, j}\right)+\operatorname{sgn}\left(p_{i, j}-p_{i, j-1}\right)\right) \times \min \left(\frac{\left|p_{i, j+1}-a_{i, j}\right|}{\Delta x+2 b_{i, j}}, \frac{\left|a_{i, j}-p_{i, j-1}\right|}{\Delta x-2 b_{i, j}}\right) .
\end{aligned}
$$

The velocity can be treated like $p_{2}$, with an additional condition linked to the CFL limitation:

$$
D_{u_{j}}=\frac{1}{2}\left(\operatorname{sgn}\left(u_{j+1}^{n}-u_{j}^{n}\right)+\operatorname{sgn}\left(u_{j}^{n}-u_{j-1}^{n}\right)\right) \times \min \left(\frac{\left|u_{j+1}^{n}-u_{j}^{n}\right|}{\Delta x+2 b_{u, j}}, \frac{\left|u_{j}^{n}-u_{j-1}^{n}\right|}{\Delta x-2 b_{u, j}}, \frac{1}{\Delta t}\right),
$$




$$
D_{v_{j}}=\frac{1}{2}\left(\operatorname{sgn}\left(v_{j+1}^{n}-v_{j}^{n}\right)+\operatorname{sgn}\left(v_{j}^{n}-v_{j-1}^{n}\right)\right) \times \min \left(\frac{\left|v_{j+1}^{n}-v_{j}^{n}\right|}{\Delta x+2 b_{v, j}}, \frac{\left|v_{j}^{n}-v_{j-1}^{n}\right|}{\Delta x-2 b_{v, j}}, \frac{1}{\Delta t}\right) .
$$

Let us remark that for aerosols, a simpler reconstruction can be used for the velocity $u$ which is the gas velocity, since no moment has to be conserved. We can then take $\overline{u_{j}}=u_{j}^{n}$ and $D_{u_{j}}$ can be a classical slope limiter.

\subsubsection{Fluxes computation}

Then we can proceed with the evaluation of the fluxes:

$$
\left(\begin{array}{l}
\boldsymbol{F}_{j+1 / 2}^{+} \\
\boldsymbol{G}_{j+1 / 2}^{+}
\end{array}\right)=\frac{1}{\Delta t} \int_{x_{j+1 / 2}^{L}}^{x_{j+1 / 2}} m_{0}(x)\left(\begin{array}{l}
1 \\
p_{1} \\
p_{1}\left[\left(1-p_{1}\right) p_{2}+p_{1}\right] \\
p_{1}\left\{\left(1-p_{1}\right)\left(1-p_{2}\right) p_{2} p_{3}+\left[\left(1-p_{1}\right) p_{2}+p_{1}\right]^{2}\right\} \\
p_{1} u \\
p_{1} v
\end{array}\right)(x) \mathrm{d} x
$$

where $x_{j+1 / 2}^{L}$ is the abscissa of the last droplets reaching $x_{j+1 / 2}$ at $\Delta t$.

$$
x_{j+1 / 2}^{L}=x_{j+1 / 2}-\Delta t \frac{\left(\bar{u}_{j}+\frac{\Delta x}{2} D_{u_{j}}\right)_{+}}{1+\Delta t D_{u_{j}}} .
$$

Similarly,

$$
\left(\begin{array}{l}
\boldsymbol{F}_{j+1 / 2}^{-} \\
\boldsymbol{G}_{j+1 / 2}^{-}
\end{array}\right)=-\frac{1}{\Delta t} \int_{x_{j+1 / 2}}^{x_{j+1 / 2}^{R}} m_{0}(x)\left(\begin{array}{l}
1 \\
p_{1} \\
p_{1}\left[\left(1-p_{1}\right) p_{2}+p_{1}\right] \\
p_{1}\left\{\left(1-p_{1}\right)\left(1-p_{2}\right) p_{2} p_{3}+\left[\left(1-p_{1}\right) p_{2}+p_{1}\right]^{2}\right\} \\
p_{1} u \\
p_{1} v
\end{array}\right)(x) \mathrm{d} x
$$

with

$$
x_{j+1 / 2}^{R}=x_{j+1 / 2}-\Delta t \frac{\left(\overline{u_{j+1}}-\frac{\Delta x}{2} D_{u_{j+1}}\right)_{-}}{1+\Delta t D_{u_{j+1}}} .
$$

This kinetic scheme, using the property of the canonical moments, preserves the moment space directly, without the use of an additional projection algorithm. Besides, since we want to couple it to a scheme for evaporation which will reconstruct from the moments the most probable distribution using the maximization of entropy [37], it is essential that the advection scheme should be well-behaved and preserves realizability inside the moment space. We summarize main characteristics of the evaporation scheme in the next section.

\section{Evaporation model and associated numerical scheme}

In the context of an operator splitting algorithm, each operator of system (5) is resolved separately. For the evaporation, a set of ODE system, one for each cell of the mesh, is obtained:

$$
\left\{\begin{aligned}
\mathrm{d}_{t} m_{0} & =-K n(S=0), \\
\mathrm{d}_{t} m_{1} & =-K m_{0}, \\
& \vdots \\
\mathrm{d}_{t} m_{N} & =-N K m_{N-1},
\end{aligned}\right.
$$

the evaporation coefficient being constant, which is the macroscopic equivalent of the kinetic equation :

$$
\partial_{t} n-\partial_{S}(K n)=0
$$

System (50) has to be closed. Indeed we have to estimate a term $-\left.K \tilde{n}\right|_{S=0}\left(m_{0}, m_{1}, m_{2}, m_{3}\right)$, representing the disappearing flux of droplets at time $t$, is a point-wise value of the NDF which has to be 
reconstructed from the data of its first $N$ moments. Achieving this amounts to solve the finite Hausdorff moment problem [12] and is done using an Entropy Maximization [39] whereas the corresponding NDF is denoted $n_{M E}(t, S)$. When it comes to the numerical resolution, pluging system (50) in a standard ODE like solver leads to serious stability problems. A robust and accurate kinetic scheme was developed in [37] which preserves the moment space. Moreover, it has been generalized to an arbitrary evaporation law, depending on the droplet size $S$ and on $t$ (through the dependance on the gas variables) [37]. We summarize herein its main features in the case of a constant evaporation rate. The solution used is to consider an integrated version in time of system (50):

$$
\exp (K \Delta t \mathbf{A}) \mathcal{M}_{N}^{n+1}=\mathcal{M}_{N}^{n}-\mathbf{\Phi}^{-},
$$

where

$$
\mathcal{M}_{N}^{n}=\left(\begin{array}{c}
m_{0} \\
\vdots \\
m_{N}
\end{array}\right)\left(t_{n}\right), \quad \mathbf{A}=\left[\begin{array}{ccccc}
0 & & & & 0 \\
1 & 0 & & & \\
& 2 & \ddots & & \\
& & \ddots & \ddots & \\
0 & & & N & 0
\end{array}\right], \quad \boldsymbol{\Phi}^{-}=\int_{0}^{K \Delta t} n\left(t_{n}, \alpha\right)\left[\begin{array}{c}
1 \\
\alpha \\
\vdots \\
\alpha^{N}
\end{array}\right] \mathrm{d} \alpha
$$

the form of the flux being found using the analytical solution of the kinetic equation (51), in the context of kinetic schemes. This integral form decouples pure transport with no flux from the evolution of the moments through the fluxes.

For the pure transport in size phase space part, a last ingredient is introduced: the computation of a quadrature of the moment vector $\mathcal{M}_{N}\left(N=2 n_{a}-1\right.$ being odd $)$. Indeed, there exist a unique vector of weight $\left(\omega_{i}\right)_{i \leq n_{a}}$ and a unique vector of abscissas $\left(\mathcal{S}_{i}\right)_{i \leq n_{a}}$ such that $m_{k}=\sum_{i=1}^{n_{a}} \omega_{i} \mathcal{S}_{i}^{k}$ for each $k=0, \ldots, N$. The following formula shows that evaporation, when the flux at zero size is zero, is only a translation of the abscissas and such an approach can be seen as equivalent to DQMOM [19]):

$$
\exp (K \Delta t \mathbf{A})\left(\begin{array}{c}
\sum_{i=1}^{n_{a}} \omega_{i}\left(t_{n}\right) \\
\sum_{i=1}^{n_{a}} \omega_{i}\left(t_{n}\right) \mathcal{S}_{i}\left(t_{n}\right) \\
\vdots \\
\sum_{i=1}^{n_{a}} \omega_{i}\left(t_{n}\right) \mathcal{S}_{i}^{N}\left(t_{n}\right)
\end{array}\right)=\left(\begin{array}{c}
\sum_{i=1}^{n_{a}} \omega_{i}\left(t_{n}\right) \\
\sum_{i=1}^{n_{a}} \omega_{i}\left(t_{n}\right)\left(\mathcal{S}_{i}\left(t_{n}\right)+\Delta t K\right) \\
\vdots \\
\sum_{i=1}^{n_{a}} \omega_{i}\left(t_{n}\right)\left(\mathcal{S}_{i}\left(t_{n}\right)+\Delta t K\right)^{N}
\end{array}\right)
$$

The resulting algorithm is then [37]:

1. Using the Entropy Maximization, we provide a reconstruction $n_{M E}\left(t_{n},.\right)$ of the distribution from its moments $\mathcal{M}_{N}^{n}$ and flux $\boldsymbol{\Phi}^{-}$is computed from this reconstruction.

2. The weights $\omega_{i}$ and the abscissas $\mathcal{S}_{i}$ corresponding to the moment vector $\mathcal{M}_{N}^{n}-\boldsymbol{\Phi}^{-}$are computed using the QD algorithm [12].

3. The moments $\mathcal{M}_{N}^{n+1}$ corresponding to the weights $\omega_{i}$ and the abscissas $\mathcal{S}_{i}-K \Delta t$ are computed.

This algorithm is valid for a CFL-like condition $K \Delta t<1$.

\section{From numerical validation to accuracy and performance assessment}

This section is devoted to three representative test-cases, with increasing difficulties, to check the robustness, the accuracy and computational efficiency of the proposed numerical scheme and algorithm. The first series of test-cases is one-dimensional. First, the transport of an aerosol in a constant velocity field provides a first level of validation and an evaluation the method accuracy. Then, a spray convection case leading to the formation of a $\delta$-shock assesses the method robustness. Finally, a complete order accuracy study of the convection of a smooth profile shows that the method is second order in space and time.

In a second test-case, a cloud of inertial droplets evolves in a two-dimensional Taylor-Green configuration for the gaseous velocity field. This case study allows us to prove the computational efficiency and 
the good behavior of the scheme. Finally, an injection of a polydisperse spray in an unsteady gaseous flow field constituted of a weakly turbulent free jet is investigated. Such a case, while still an academic testcase, is close to more realistic configurations and shows the robustness of the scheme in a more complex environment. We provide a detailed comparison with results given by the multi-fluid model and assess the efficiency of the proposed approach. Let us underline that such a configuration has been introduced in the Ph.D. Thesis of Hicham Meftah [40] at CORIA and that we have compared the Eulerian multi-fluid approach to a reference Lagrangian simulation in $[9,8]$. The conclusions of this study was that the fuel mass fraction issued from evaporation was extremely well predicted by the multi-fluid approach, thus making it also a reference solution as far as the present method is concerned.

\subsection{Validation by comparison with analytical solutions for $1 D$ aerosol and spray transport}
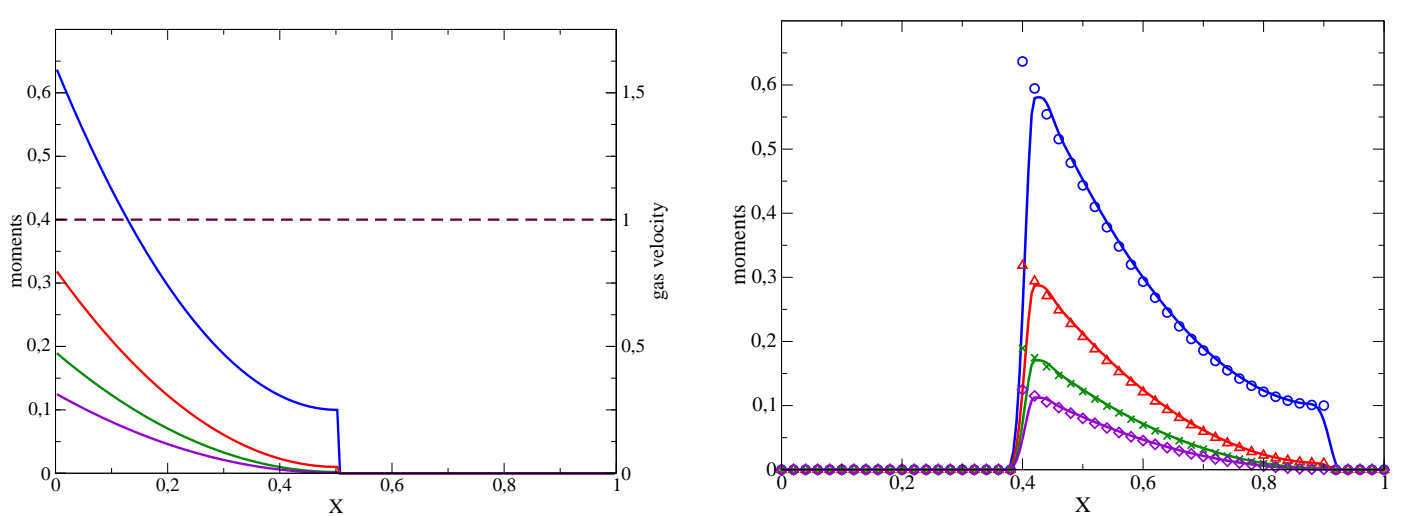

Figure 1: Evolution of aerosol droplets calculated with the EMSM model in a constant velocity field. (left) Initial condition; the plain curves represent, with decreasing ordering in terms of value, the four first moments from the $0^{t h}$ order (highest) to the $3^{r d}$ (lowest). The plain curves are scaled by the left Y-axis and the dashed curve representing the velocity field, is scaled by the right Y-axis. (right) Solution at time $t=0.4$ compared to the analytical solution of the problem, represented by marquers.

We first simulate the transport at constant velocity of a polydisperse aerosol. The gas velocity is taken as constant and equal to one everywhere and an aerosol is initially present in half of the domain $[0,1]$, and the boundary conditions are periodic. Its size distribution has initially a concave shape at $x=0$ (part of the sinusoid) and a convex one at $x=0.5$ (decreasing exponential). It evolves continuously between these two points:

$$
n(t=0, x, S)= \begin{cases}\lambda(x) \sin (\pi S)+(1-\lambda(x)) \exp (-10 S), & x \leq 0.5 \\ 0, & x>0.5\end{cases}
$$

where $\lambda(x)=4(0.5-x)^{2}$. The first four size moments of this initial distribution are displayed on Fig. 1-left. The constant velocity field is also represented. According to this velocity field, we expect a translation of all the moments at velocity one. That is indeed what we obtain in Fig. 1-right, the numerical simulation being performed in a 200 cell grid with a convective CFL number equal to one. The first conclusion, that we can draw from this simple test-case is that only numerical diffusion alters the accuracy of the moment resolution. We must highlight the fact that this is done without an extra projection algorithm, yet yields a rather high level of precision.

In the second test-case, a quantitative comparison with an analytical solution of the transport of an evaporating spray of ballistic droplets is performed. For this problem, we choose the same size distribution as before, but now the droplets have their own velocity, initiated by:

$$
u(x)= \begin{cases}0.5, & x \leq 0.25 \\ 2, & x>0.25\end{cases}
$$



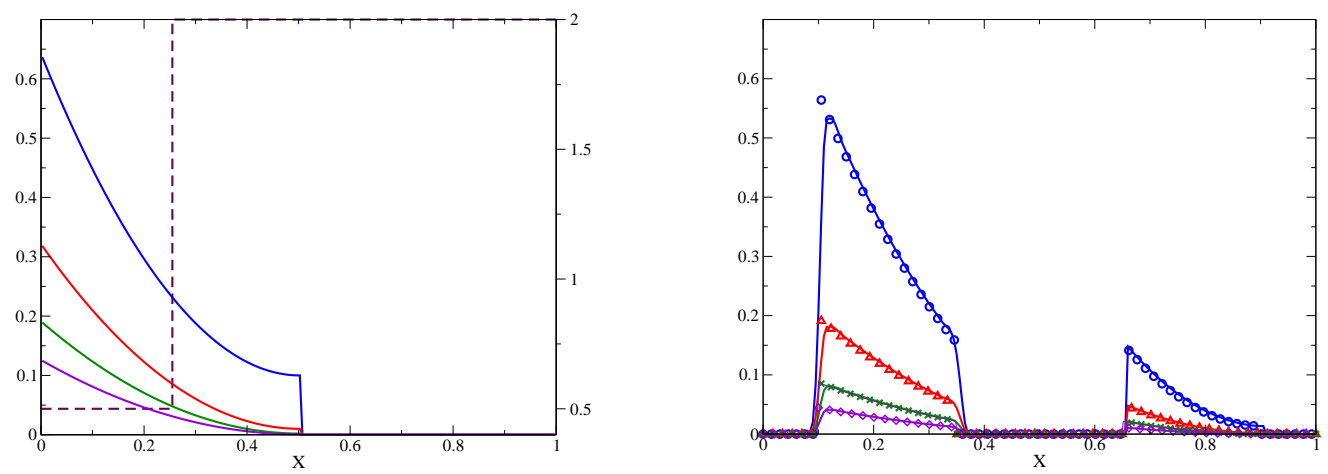

Figure 2: Evolution of a spray in a discontinuous velocity field, calculated with the EMSM model, compared to the analytical solution of the problem. (left) Initial condition; the plain curves represent, with decreasing ordering in terms of value, the four first moments from the $0^{t h}$ order (highest) to the $3^{\text {rd }}$ (lowest). The plain curves are scaled by the left Y-axis and the dashed curve representing the velocity field, is scaled by the right Y-axis. (right) Solution at $t=0.2$ compared to the analytical solution of the problem, represented by marquers.

The initial conditions are displayed in Fig. 2-left. As we consider ballistic droplets, their dynamics is not influenced by the gas. Besides, the droplets are assumed to be evaporated at rate $K=1$. The spatial velocity discontinuity makes the droplet cloud spread into two separate clouds with two distinct velocities. This configuration shows the ability of the method to handle the vacuum zone generated by the separation of the clouds. Moreover, because of periodic boundary conditions, the faster cloud will catch up with the slower one once it has re-entered the domain on the left side. Figure 2-right displays the four analytical size moments, and the size moments given by the calculation at the time $t=0.2$, performed in a 200 cell grid with a convective CFL equal to one. Figure 3 displays the four moments at times $t=0.4$. First, the numerical solution perfectly matches the analytical one, second the moment space is automatically preserved. The initial distribution breaks into two parts. Vacuum is created at the initial velocity discontinuity. At $\mathrm{t}=0.4$, the fastest portion catches up with the slower one. As we consider

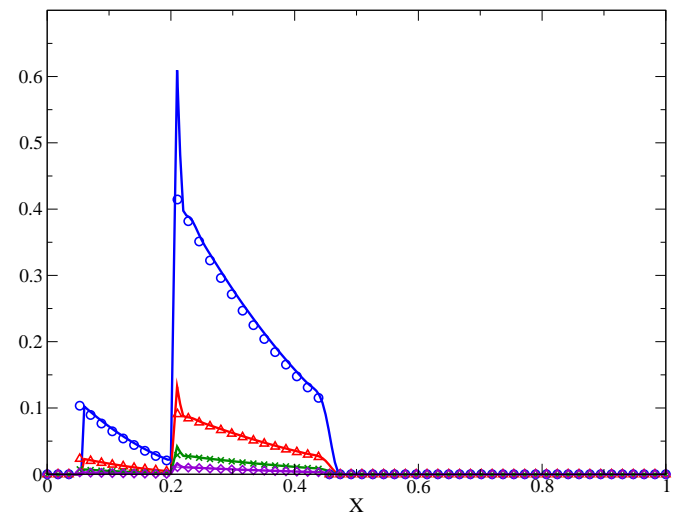

Figure 3: Evolution of a spray in a discontinuous velocity field calculated with the EMSM model, compared to the analytical solution of the problem. Solution at time $t=0.4$ compared to the analytical solution of the problem represented by marquers.

a pressureless gas formalism for the droplets, nothing prevents the droplets from accumulating. However, it must be kept in mind that the collision probability is negligible, to that the real physical solution would result in a crossing of the clouds without interaction such as presented through the analytical solution. Simulating jet crossing is an issue in Eulerian models and and new methods have been recently designed in the literature and we refer to $[17,5,27,48]$ for references. Let us mention that the coupling of the present algorithm with particle trajectory crossing can be found in the review paper [36]. The conclusion of that 
test-case study reveals that our numerical approach is accurate relative to transport and evaporation and still preserves the realizability condition even in the presence of singularity formation.

\subsection{Order accuracy study}

An order accuracy study is performed for the first and second order kinetic schemes for pure advection of a moment set. The initial size distribution has the following profile:

$$
n(t=0, x, S)= \begin{cases}\alpha(x) \frac{\alpha(x) \sin ((1-x) \pi S)+(1-\alpha(x)) \exp (-10(1-x) S)}{\int_{0}^{1} \alpha(x) \sin ((1-x) \pi S)+(1-\alpha(x)) \exp (-10(1-x) S) \mathrm{d} S}, & x \leq 0.5 \\ 0, & x>0.5\end{cases}
$$

where $\alpha(x)=\exp -\frac{(0.25-x)^{2}}{\sigma^{2}}$, where $\sigma=0.12$. Such a spatial and size distribution with nonlinear coupling can be considered as general enough for an accuracy study in order to quantify the order of convergence of the proposed scheme. The velocity field is initiated as shown in Fig. 4-right. It writes:

$$
u(t=0, x)= \begin{cases}1-x, & x \leq 0.5 \\ 0, & x>0.5\end{cases}
$$

The negative slope of its linear profile leads to a compression of the moment field at the rate given by solving the equation $d_{t} m / m=-\partial_{x} u=0.5 /(0.5-0.5 t)$. As the final computational time is 0.5 , the final value of the moments is $m_{k}(t=0.5, x)=2 m_{k}\left(t=0, x_{0}\right)$, where $x$ is the point, at $t=0.5$, of the characteristic containing the point $\left(t=0, x_{0}\right)$. The computation is run with $C F L=1$.

Two types of results are given. The first type gives the final profile of the moments, comparing the results for the first and second order schemes, for the 100 cell grid, with the analytical results, see Fig. 5. This clearly shows the gain of accuracy brought by second order scheme in comparison to first order scheme. Grid convergence studies for the first and second order advection schemes are presented in Fig. 6. For both the schemes, the profile of $m_{0}$ obtained using different grid resolutions is compared to the analytical solution. Four different uniform grids have been considered with the number of cells equal to 25,50,100 and 200. Figure 6-left shows grid convergence for the first order scheme, and Fig. 6-right shows grid convergence for the second order scheme. A remarkable result is the low numerical diffusion level of the second order scheme. Generally, numerical diffusion has two origins: the reconstruction step and the flux time integration computation at the cell interfaces in cell center finite volume schemes. The proposed second order kinetic scheme involves an exact time integration of the flux, preventing any numerical diffusion to occur at this level. This comes from the fact that the velocities of the waves of the system are explicitly known at time $t_{n}$ since they are not modified by any acoustic effect: this result is specific to the pressureless gas formalism considered here.

Table 1 and 2 show the numerical order results for both schemes and provide detailed results for the four moments. The mean order is the slope of the straight line given by a linear regression of the four error results per moment. For the first order scheme, the numerical order is lower than one (0.76). Such a result is rather classical for such a class of finite volume method (see [48] for example). For the second order scheme, the numerical order for $m_{0}$ is little lower than two (1.82). Second order is reached for all other moments. Figure 7 displays the order curves for the first and second order schemes, highlighting the precision difference between both methods and showing the high level of accuracy reached by the second order method. Such results can be considered as very satisfying compared to other existing methods.

\begin{tabular}{|c|cccc|c|}
\hline Grid size & 25 & 50 & 100 & 200 & Order \\
\hline$m_{0}$ & 0.5176 & 0.3306 & 0.1932 & 0.1077 & 0.76 \\
\hline$m_{1}$ & 0.5534 & 0.3673 & 0.2182 & 0.1214 & 0.74 \\
\hline$m_{2}$ & 0.5658 & 0.3764 & 0.2247 & 0.1250 & 0.73 \\
\hline$m_{3}$ & 0.5686 & 0.3802 & 0.2274 & 0.1268 & 0.72 \\
\hline
\end{tabular}

Table 1: $L_{1}$ error and order of accuracy of the first order kinetic scheme. 

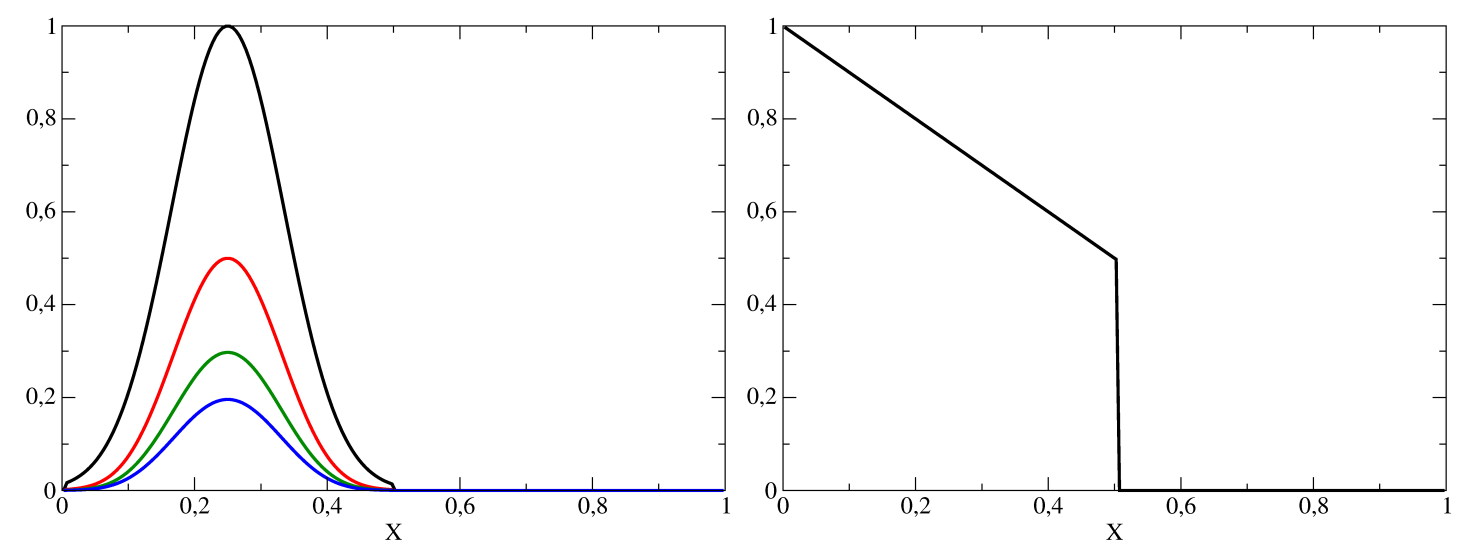

Figure 4: Order accuracy study. Initial conditions. Left: Initial moment fields. The plain curves represent, with decreasing ordering in terms of value, the four first moments from the $0^{\text {th }}$ order (highest) to the $3^{\text {rd }}$ (lowest) Right: Initial velocity field.
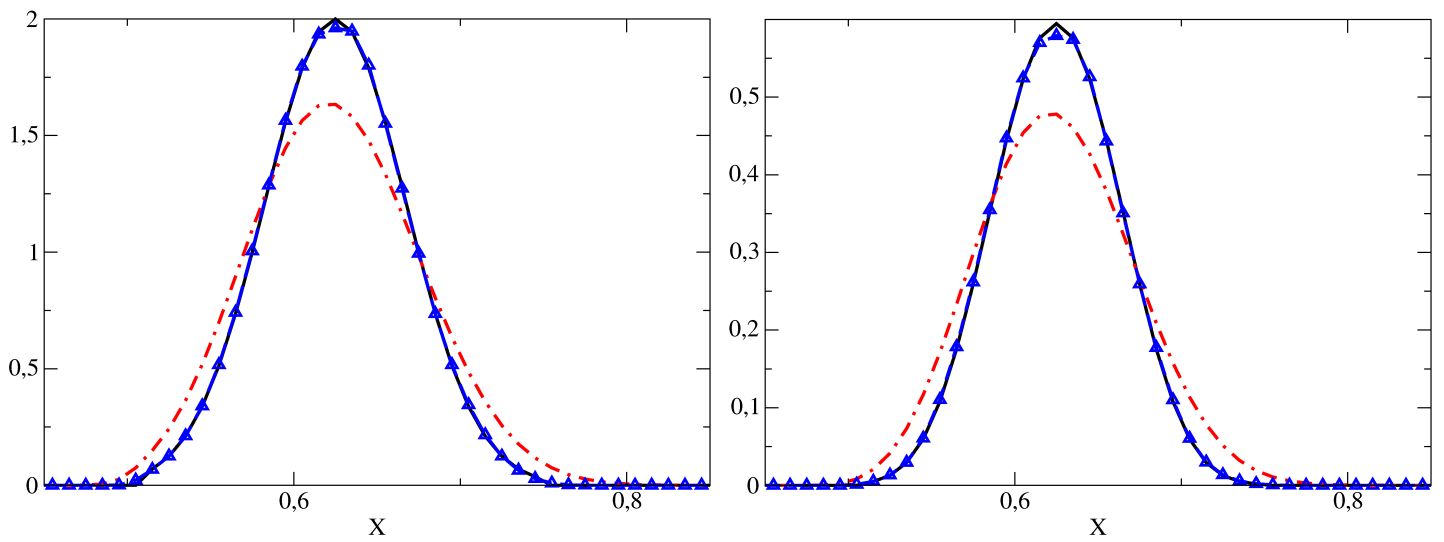

Figure 5: Order accuracy study. Moment profile for the final time, with a 100 cells grid. Comparison of the first and second order scheme with the analytical solution. The plain black curve represents the analytical solution, the dashed blue curve with triangles and the dotted-dashed red curve represent the numerical solution respectively for second and first order scheme. Left: $m_{0}$. Right: $m_{2}$.
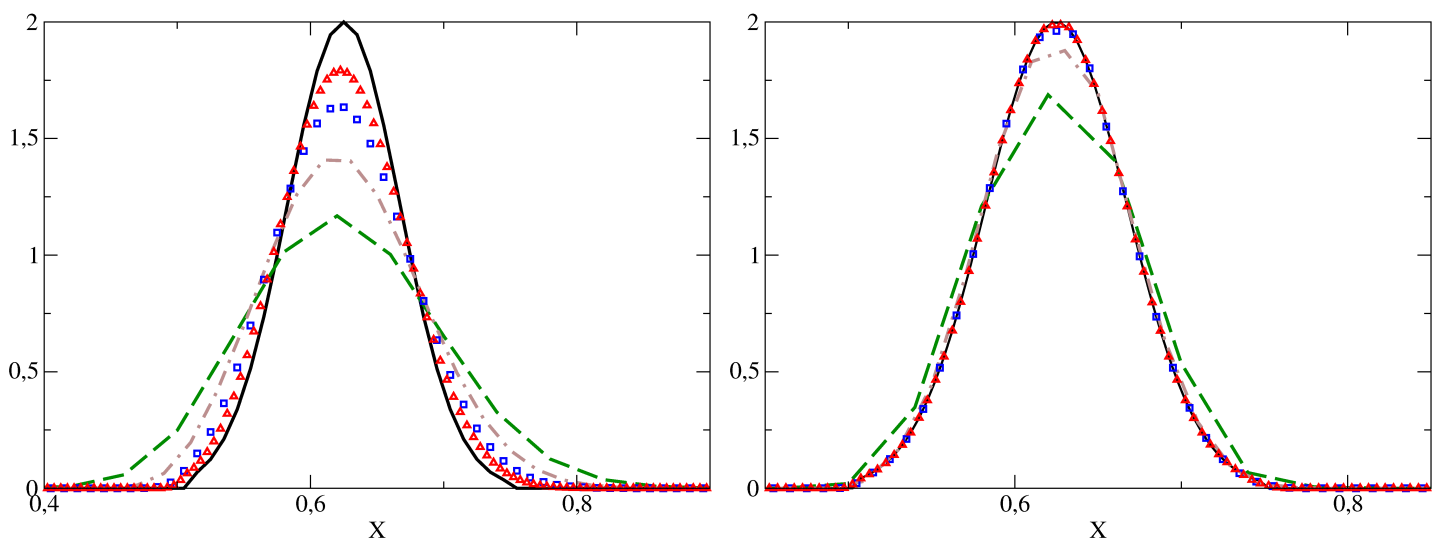

Figure 6: Order accuracy study. Profile of $m_{0}$ for the final time. Results are displayed for 200 cells (red triangles), 100 cells (blue squares), 50 (dotted-dashed brown curve), 25 (dashed green curve). Left: first order. Right: second order. 


\begin{tabular}{|c|cccc|c|}
\hline Grid size & 25 & 50 & 100 & 200 & Order \\
\hline$m_{0}$ & 0.1216 & 0.0318 & 0.0079 & 0.0028 & 1.82 \\
\hline$m_{1}$ & 0.1667 & 0.0436 & 0.0093 & 0.0025 & 2.04 \\
\hline$m_{2}$ & 0.1798 & 0.0478 & 0.0102 & 0.0028 & 2.01 \\
\hline$m_{3}$ & 0.1864 & 0.0499 & 0.0110 & 0.0034 & 1.95 \\
\hline
\end{tabular}

Table 2: $L_{1}$ error and order of accuracy of the second order kinetic scheme.
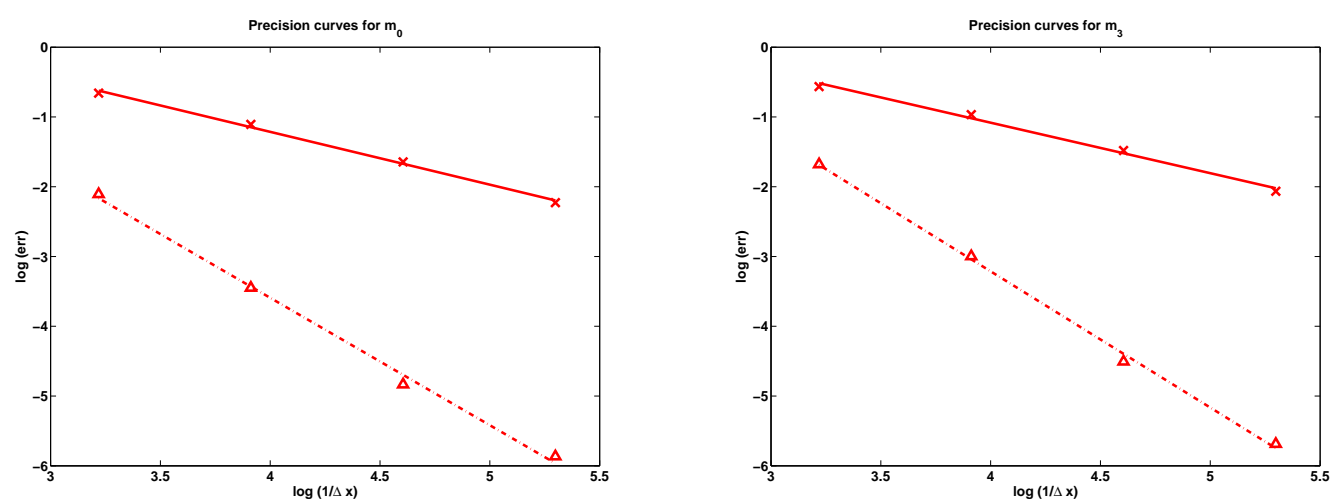

Figure 7: Order accuracy study. Error curves with respect to grid refinement in logarithmic scale. The solid line represents the first order scheme, the dotted-dashed represents the second order scheme. The symbols represents the logarithm of the error given by the computation. Left: results for $m_{0}$; Right: results for $m_{3}$.

\subsection{Comparison with other methods in literature}

The ground difference between the scheme developed here for the advection of a moment set and the type of schemes presented in [50] and [38] is that the moment set integrity is preserved by the scheme itself. Thus, no additional numerical test or additional algorithm needs to be performed. This difference is sufficiently important so that no further numerical comparisons are relevant.

In [48], Vikas et. al design a second order quadrature-based scheme for the transport of a velocity moment vector. Whereas they are mainly interested in designing a second order scheme for the transport of velocity moment vectors, their ideas can be applied for the transport of size moments. Let us remark first that, in this article, we tackle the issue of the transport of an evaporating spray. Indeed, evaporation is an essential process to take into account in the description of spray dynamics. Therefore, if the main achievement of this paper concerns a second-order advection scheme preserving the moment set integrity, it is also very important that this scheme can be coupled to the evaporation solver presented in Section 4. However, even if it could be envisioned to couple, through an operator splitting, our evaporation algorithm to the transport algorithm proposed in [48], we still want to make sure that the accuracy is sufficient in order to obtain a global scheme that is competitive with multi-fluid models and methods. Thus, for pure transport, it is interesting to perform a detailed accuracy comparison between the two schemes on the previous test case. Results are detailed in Appendix 7.B which allow us to conclude on the high level of accuracy of our scheme as well as on the novelty of the present approach.

\subsection{Two dimensional dynamics of a droplet cloud in Taylor-Green vortices}

We test our high order moment method in a multi-dimensional configuration with vortices: the 2-D Taylor-Green vortex flow, a steady solution of the inviscid incompressible Euler equations. The gaseous non-dimensional velocity field is then given by $u_{g, x}=\sin (2 \pi x) \cos (2 \pi y)$ for its horizontal component and $u_{g, y}=-\cos (2 \pi x) \sin (2 \pi y)$ for its vertical one, with $(x, y) \in[0,1]^{2}$ and with periodic boundary conditions. The structure of the flow field is presented in Fig. 8-left through the velocity vectors. The initialization of the spray corresponds to a motionless cloud overlapping two different vortex areas, which will be dragged by the gas. Figure 8-right shows the initial spatial mass distribution provided by a 

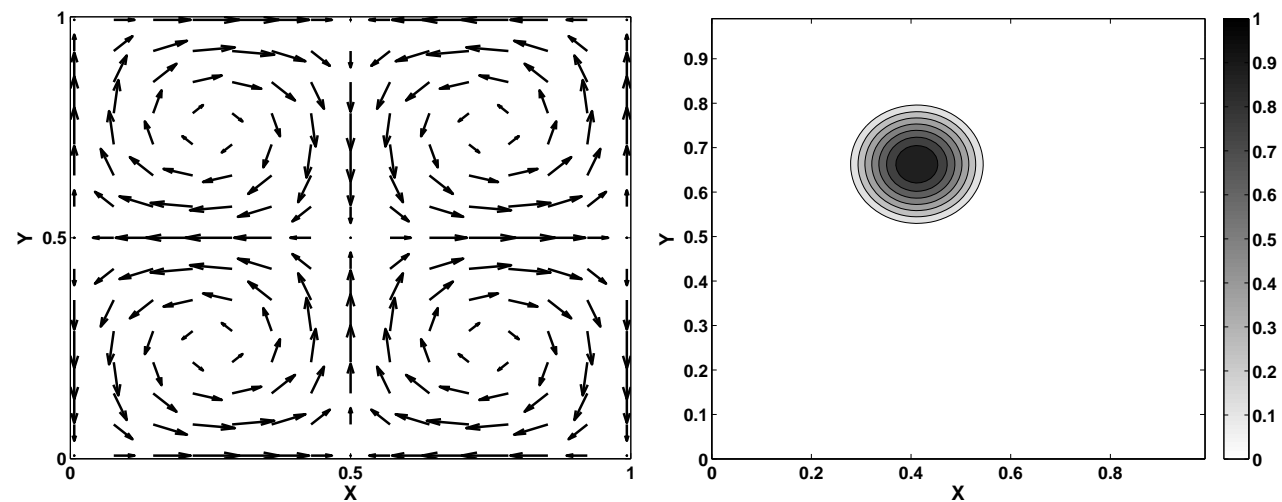

Figure 8: (left) Taylor-Green configuration for the gas vorticity field, (right) Initial condition for the droplets, composed of a motionless cloud. The droplet mass is represented, which is reconstructed from the first four size moments: $\left(m_{0}, m_{1}, m_{2}, m_{3}\right)$.

cardinal sinus function. The size distribution of the droplets is initially uniform, with a NDF as well the corresponding mass distribution function represented in Fig. 9.
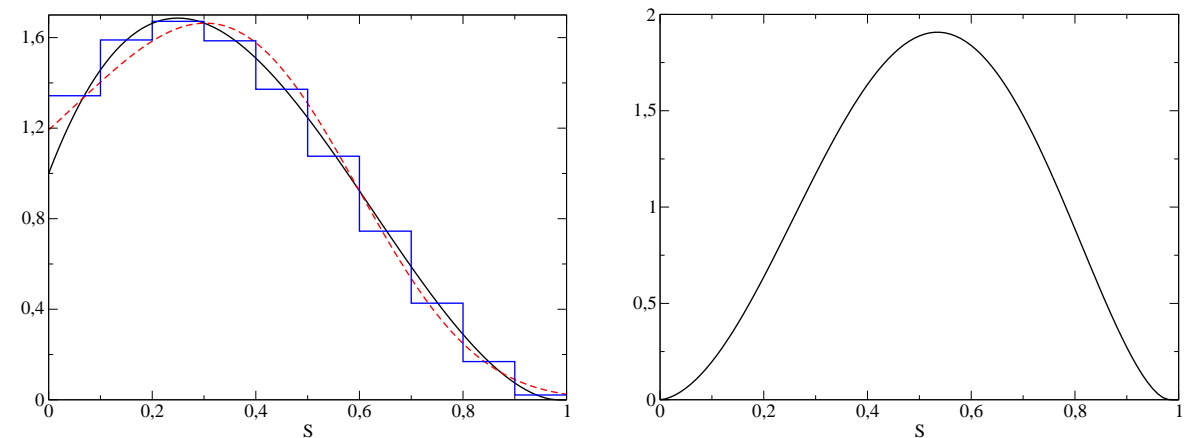

Figure 9: (left) Initial size distribution for the droplets (solid line), its reconstruction by the Entropy Maximization from its first 4 moments (dashed line) and initial mass distribution for the multi-fluid method (piece constant function). (right) Corresponding mass distribution. 

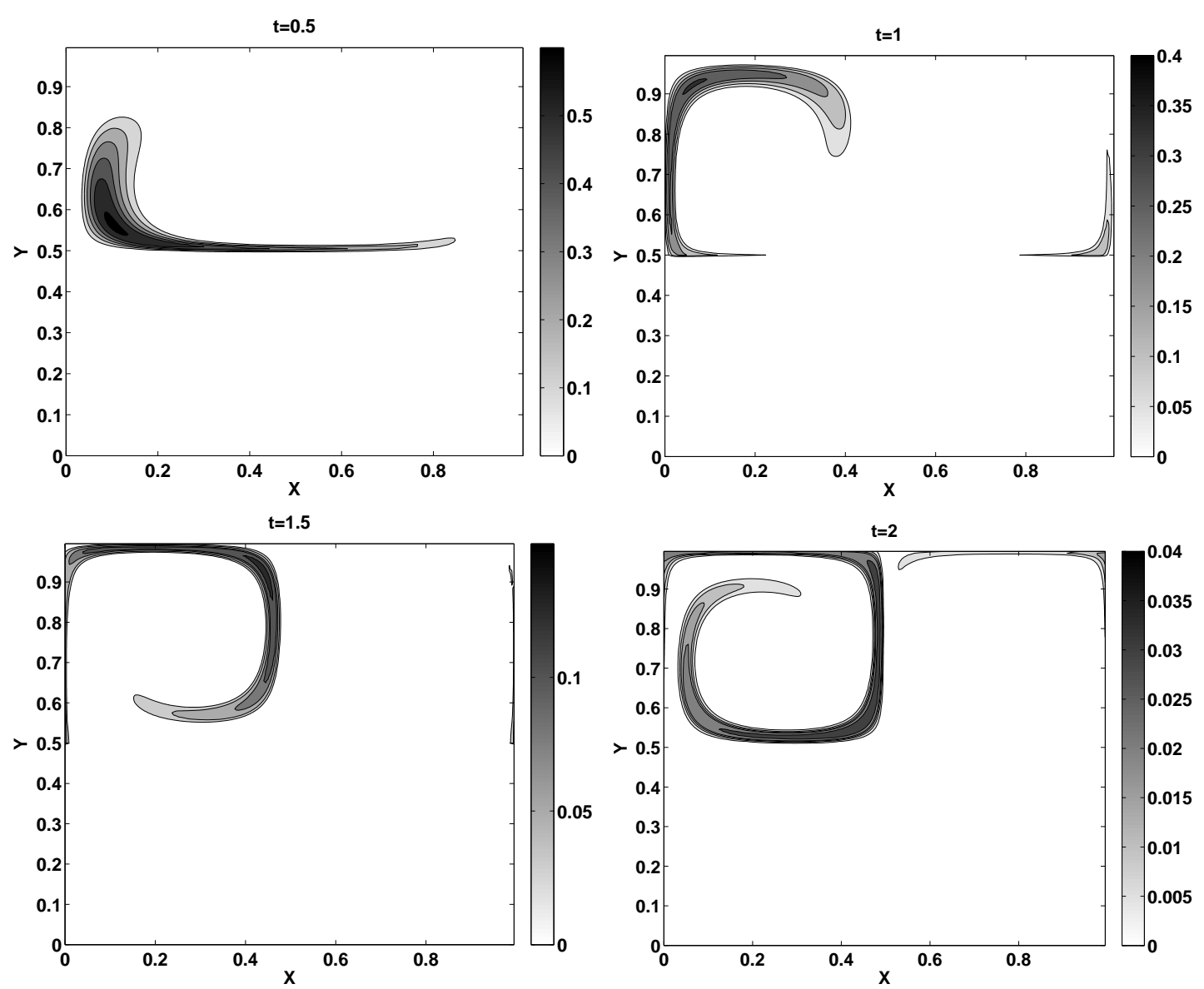

Figure 10: Results for spray dynamics dragged by the gas field made of Taylor-Green vortices, computed with the EMSM model. Droplet mass at time $t=0.5$ (top-left), $t=1$ (top-right), $t=1.5$ (bottom-left), $t=2$ (bottom-right). The computation is carried out in a $200 \times 200$ cell grid.

Two sets of computations are presented, one for the EMSM model and the other one for the multifluid model with ten sections. Both are done till time $t=2$, corresponding to one eddy turn over time. Moreover, we have chosen a $200 \times 200$ grid cell, to ensure that we have a sufficiently detailed description of the field and, as in the earlier cases, the CFL number is equal to one. The results of the multi-fluid model are used as the reference to validate our method. The field compared between the two results is the droplet mass density which is naturally solved in the multi-fluid model, whereas it is reconstructed from the four moments $\left(m_{0}, m_{1}, m_{2}, m_{3}\right)$ in the high order moment method, using the reconstruction $n_{M E}$ computed by Entropy Maximization [37].

The droplet Stokes number of the distribution, based on the typical size $m_{1} / m_{0}$, is initially 0.017 for the EMSM model, and ranges from 0.0028 to 0.045 for the multi-fluid model with ten sections. The spray evaporation rate is set as $K=0.27$. Figures 10 and 11 show the evolution of the spatial mass distribution for both computation. Due to the cloud initial location, two parts of it are dragged by two different vortices. That is why the main part of the cloud is dragged in the top-left vortex, whereas a smaller part of the cloud is dragged in the top-right vortex. From $[35,8,36]$, we know that there exists a critical value $\mathrm{St}_{c}=1 / 8 \pi(\approx 0.0398)$ which separates two regimes. For $\mathrm{St}<\mathrm{St}_{c}$, the droplets cannot escape from the Taylor-Green vortices while, for $\mathrm{St}_{2} \geq \mathrm{St}_{c}$, they are ejected out of their original vortices. This explains that, with the multi-fluid method, a part of the mass is in the bottom vortices: Stokes number under consideration goes beyond $\mathrm{St}_{c}$ for the last sections. However, this is not captured by the high order moment method since only one averaged Stokes number is considered and is lower than $\mathrm{St}_{c}$.

However, the general notice is that the level of comparison is very good. Indeed, results at $t=0.5$ 

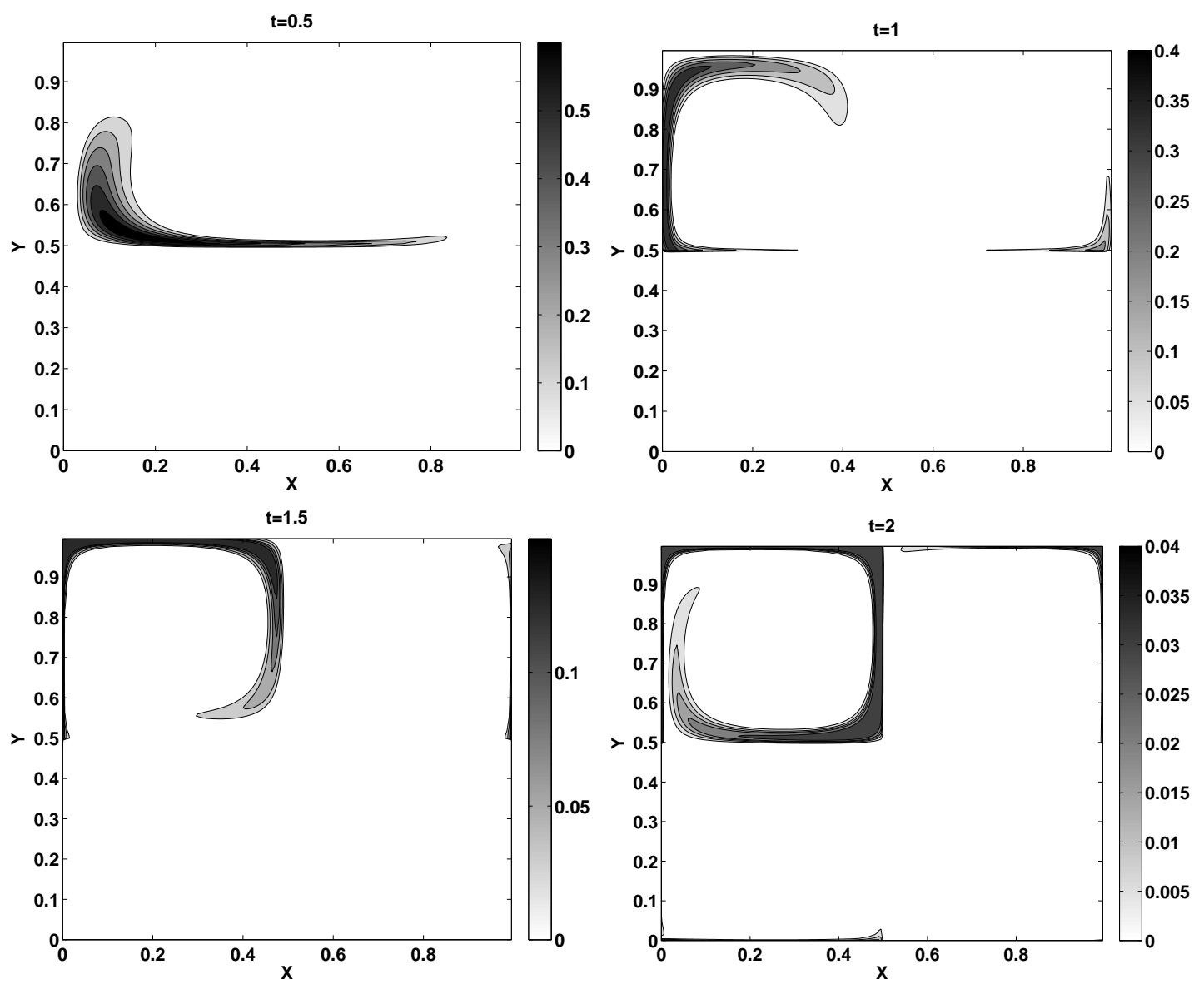

Figure 11: Results for spray dynamics dragged by the gas field made of Taylor-Green vortices, computed with the multi-fluid model for ten sections. Droplet mass at time $t=0.5$ (top-left), $t=1$ (top-right), $t=1.5$ (bottom-left), $t=2$ (bottom-right). The computation is carried out in a $100 \times 100$ cell grid.

and $t=1$ are very similar between the two methods. At time $t=1.5$ and $t=2$, the fact that the droplets are dragged faster in the case of the EMSM model comes from the computation of the drag term. In both models, the Stokes number writes $\theta \bar{S}$, where $\theta$ is defined in Eq. (2.2) and $\bar{S}$ is the average droplet surface of the distribution. In the case of the multi-fluid model, $\bar{S}=\frac{3}{5}\left(S_{i+1}^{5 / 2}-S_{i}^{5 / 2}\right) /\left(S_{i+1}^{3 / 2}-S_{i}^{3 / 2}\right)[31]$. As indicated in Fig. 9-right, the sections with the highest mass are between 0.44 and 0.6, so that $\bar{S}$ is approximately 0.52 . whereas for the EMSM model $\bar{S}=m_{1} / m_{0}=0,3487$. Since the multi-fluid model considers the dynamics of ten sections, it better resolves the spray dynamics conditioned by size than the EMSM model that only describes the droplet mean velocity. Nevertheless, in order to assess the accuracy of the models in terms of mean dynamics of the droplets, we have performed some studies on the evolution of the mean droplet size through evaporation. First, Fig. 12-left displays the evolution of the droplet mass through evaporation of a motionless cloud. It can be concluded that the multi-fluid model, with ten sections, is not as accurate as the EMSM model . Further comparisons and conclusions can be found in [37]. In a second study, the evolution of the mean particle size through evaporation, given by both the models, are compared. It can be seen in Fig. 12-right that the EMSM model is more accurate than the multi-fluid model in assessing the dynamical value of $m_{1} / m_{0}$. That means that, the term of mean drag, and thus the mean droplet velocity is better solved by the EMSM model than the multi-fluid model. Remarkably enough, even with 40 sections, the multi-fluid model does not give as good results as the EMSM model. The conclusion to be drawn from the previous elements is that, even if the multi-fluid model is able to capture the details of the dynamics conditioned on droplet size, the mean dynamics, 
for droplets below the critical Stokes number, is very well captured, even better captured, compared to the multi-fluid model. On the other hand, let us recall that the accuracy of the description of the size distribution function is better in the EMSM model for the evaporation process [37].

Finally we would like to emphasize the fact that, to solve this problem, only six equations are solved in our model, whereas three equations per section are solved in the multi-fluid model, for a total of thirty equations, in two space dimensions.
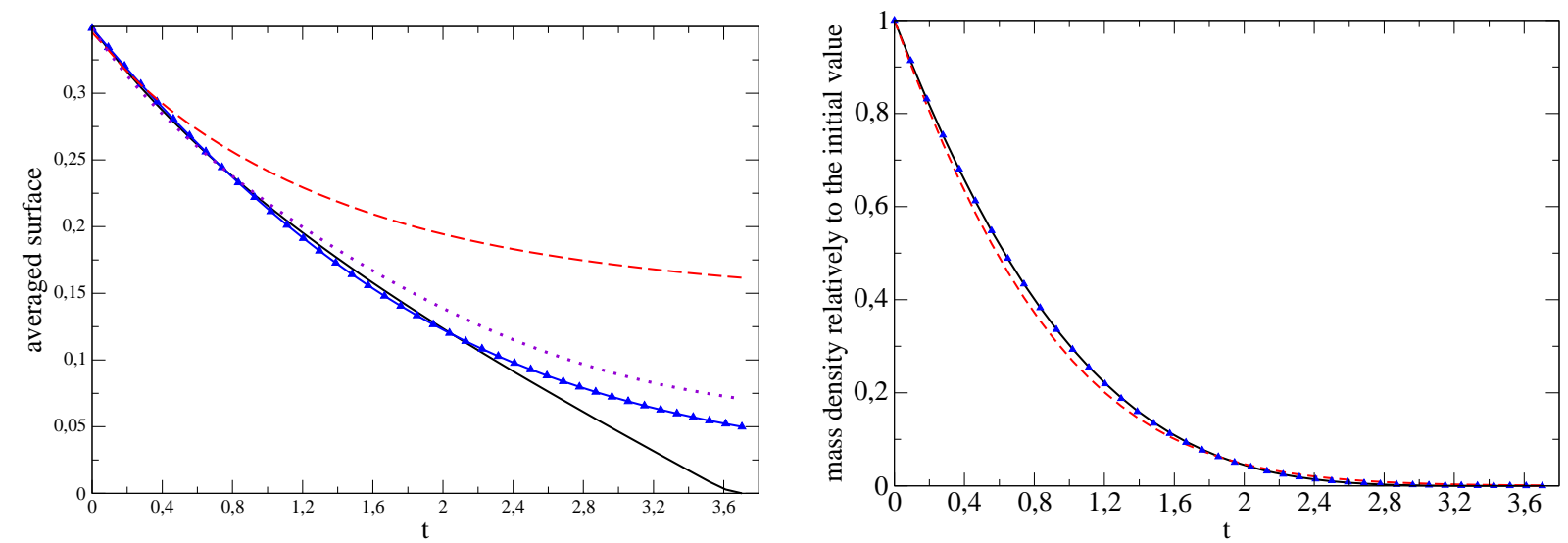

Figure 12: (Left) Evolution of the droplet mass of a motionless cloud through evaporation with a $d^{2}$ law, and comparison with the analytical solution. (Right) Evolution of the mean droplet size through evaporation, and comparison with the analytical solution. Black solid curve: analytical solution; triangles: EMSM model; dashed red curve: multi-fluid model with ten sections; violet dots: multi-fluid model with 40 sections.

The critical question of time efficiency is addressed in a study comparing the computation time required for the EMSM model and for the multi-fluid model with ten sections, on the configuration described above. Three different computations have been performed, first on a $50 \times 50$, then on a $100 \times 100$ and finally on a $200 \times 200$ cell grid. For each computation, the total CPU time is assessed. Moreover, in order to evaluate the relative importance of the phase space and physical space transport, the time spent in each and every routine is recorded. Table 3 displays the results of the analysis on the total computation time and the relative time spent for the resolution of transport in phase space and physical space, respectively. This analysis points out the time efficiency of the EMSM model, which is about 4 times faster than the multi-fluid method (MF). This is a real achievement, given the quality of the comparison of the results. The second result provided is that the relative importance of phase space transport decreases as the number of cells increases. Moreover, Table 4 compares the number of variables solved for each operator resolution, for both models. For phase transport, six variables must be solved in the case of the EMSM model (the four size moments and the two components of the velocity), while $n_{M E}$, the reconstructed NDF is obtained from the moments by an iterative solver. On the other hand, three variables per section, the mass (denoted $m_{3 / 2}$ as it is proportional to the size moment of order $3 / 2$ ) and the two components of the velocity, are explicitly solved in the case of the multi-fluid model, which amounts to thirty variables in total.

It gives the ratio between the computation time of the multi-fluid to the computation time of the EMSM model. Table 4 shows that the term requiring most of the CPU time is physical transport. and the multi-fluid (with ten sections) resolution takes four times as long as the resolution with the EMSM model.

Extrapolating from these results, we can legitimately expect that this ratio will become higher in three dimensions, and that our new method will be much faster than the multi-fluid model, especially since most of the computation time will be devoted to transport in physical space [20]. We also notice that the phase space transport can easily be parallelized. As a result, our new method proves to be very attractive for three-dimensional configurations. 


\begin{tabular}{|c|c|c|c|c|c|c|c|c|c|}
\hline Grid & \multicolumn{3}{|c|}{$50 \times 50$} & \multicolumn{3}{|c|}{$100 \times 100$} & \multicolumn{3}{|c|}{$200 \times 200$} \\
\hline & Phase & Physical & Total (s) & Phase & Physical & Total (s) & Phase & Physical & Total (s) \\
\hline EMSM & $49.6 \%$ & $50.4 \%$ & 52 & $43 \%$ & $57 \%$ & 318 & $34.5 \%$ & $65.5 \%$ & 2140 \\
\hline $\mathrm{MF}$ & $56.79 \%$ & $43.21 \%$ & 205 & $47.25 \%$ & $52.75 \%$ & 1269 & $38.39 \%$ & $61.61 \%$ & 8461 \\
\hline Ratio & 4.5 & 3.4 & 3.9 & 4.4 & 3.7 & 4 & 4.4 & 3.7 & 3.9 \\
\hline
\end{tabular}

Table 3: Computation time comparison between the EMSM model and the multi-fluid model (MF). Phase denotes the relative time (in pourcent of the total time) spent for phase space transport (evaporation and drag). Physical denotes the relative time (in pourcent of the total time). Total denotes the total computation time (in seconds).

\begin{tabular}{|c|c|c|c|}
\hline & EMSM & MF & Ratio time \\
\hline Phase & $m_{0}, m_{1}, m_{2}, m_{3},+n_{M E}$, & $10 \times\left(m_{3 / 2}, u, v\right)$ & \\
& $\begin{array}{c}u, v \\
=6 \text { variables }+n_{M E}\end{array}$ & $=30$ variables & 4.4 \\
\hline Physical & $m_{0}, m_{1}, m_{2}, m_{3}$, & $10 \times\left(m_{3 / 2}, u, v\right)$ & \\
& $u, v$ & $=30$ variables & 3.6 \\
\hline
\end{tabular}

Table 4: Comparison of the number of variables for the EMSM model and the multi-fluid model (MF), for phase space and physical space transport. The last column represents the approximate ratio of the computational time spent for the multi-fluid model to the time spent for high order moment method, in the phase and physical transport respectively.

\subsection{Polydisperse evaporating spray in a weakly turbulent free jet configuration}

In this third test-case the EMSM model is assessed on a 2-D Cartesian weakly turbulent free jet. A polydisperse spray is injected in the jet core with the size distribution represented in Fig. 9-left. The simulations are conducted with an academic solver, coupling the ASPHODELE solver, developed at CORIA by Julien Reveillon and collaborators [43, 44], with the Eulerian solver MUSES3D [8, 36] developed at EM2C Laboratory, using the models and the numerical scheme presented in these article. The ASPHODELE solver couples an Eulerian description of the gas phase with a Lagrangian description of the spray.

As far as the gas phase is concerned, a 2-D Cartesian low Mach number solver is used. The gas jet is computed on a $400 \times 200$ uniformly spaced grid. To destabilize the jet, turbulence is injected using the Klein method with $10 \%$ fluctuations [30]. The Reynolds number based on $U_{0}, \nu_{0}$ and $L_{0}$ is 1,000 , where $U_{0}$ is the injection velocity and $L_{0}$ is the jet width. Dimensional quantities for illustration purposes will be based on a velocity of $U_{0}=1 \mathrm{~m} / \mathrm{s}$ and $L_{0}=1.5 \times 10^{-2} \mathrm{~m}$, with typical value of $\nu_{0}=1.6 \times 10^{-5} \mathrm{~m}^{2} / \mathrm{s}$. Finally we have $\rho_{l} / \rho_{g}=580$. The gas vorticity is presented in Fig. 13.

As in the previous case studies, in order to validate the developments detailed in this article, two computations have been performed, one with our model and the second with the multi-fluid model. The efficiency of the multi-fluid model in describing polydisperse evaporating sprays has been demonstrated in $[9,27]$.

This free jet case is computed with an evaporating spray having an evaporation coefficient $K=0.07$. The droplet Stokes number is 0.275 for the EMSM model, corresponding to a diameter $d_{0}=75 \mu \mathrm{m}$, and ranges from $\mathrm{St}=0.047$ to $\mathrm{St}=0.75$ for the multi-fluid model with ten section, corresponding to diameters from $d_{0}=5 \mu \mathrm{m}$ to $d_{0}=85 \mu \mathrm{m}$. In order to correctly describe the evaporation process with the multi-fluid model, ten sections are considered, whereas only one section is considered in the EMSM model. The computation runs until $t=20$. Figure 14 displays the final mass fields for the spray. The level of comparison between the two resulting fields is very good. One can nevertheless notice a slight difference, i.e in the multi-fluid model with ten sections, the spray is less evaporated.

Since our primary interest is in combustion applications, the main objective of evaporating spray modeling is prediction of the gas-phase fuel mass fraction. Therefore we present comparisons between the gas-phase fuel mass fraction obtained from the EMSM model and multi-fluid descriptions of the spray. These simulations were once again accomplished using one-way coupling. This enables to have 


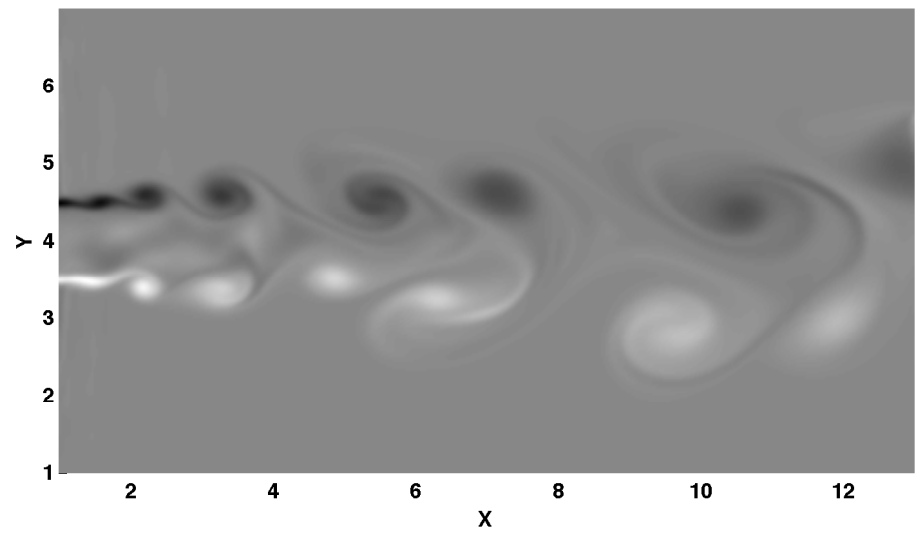

Figure 13: Free-jet configuration at time $t=20$. Gas vorticity on a $400 \times 200$ cell grid.

the exact same gaseous field for computations with both models. Therefore, the comparisons are only focused on the spray models. As a consequence, the evaporated fuel is not added as a mass source term in the gas-phase equations, but is stored in passive scalars for the gas [8]. The two fields are plotted in Fig 15. These results confirm the quality of the previous results. The present comparison, by showing clear similarity between the two models, highlights the efficiency of the EMSM model and the associated numerical schemes in describing polydisperse evaporating sprays. It has both the ability to cope with exactly zero droplet mass density and model singularities formation as well as the capability to propose an accurate Eulerian treatment of the polydisperse evaporating spray with a very limited amount of numerical diffusion.

These results are a first significant step towards combustion computations with full two-way coupling or flame dynamics in 3D such as the simulations presented in $[22,20]$.
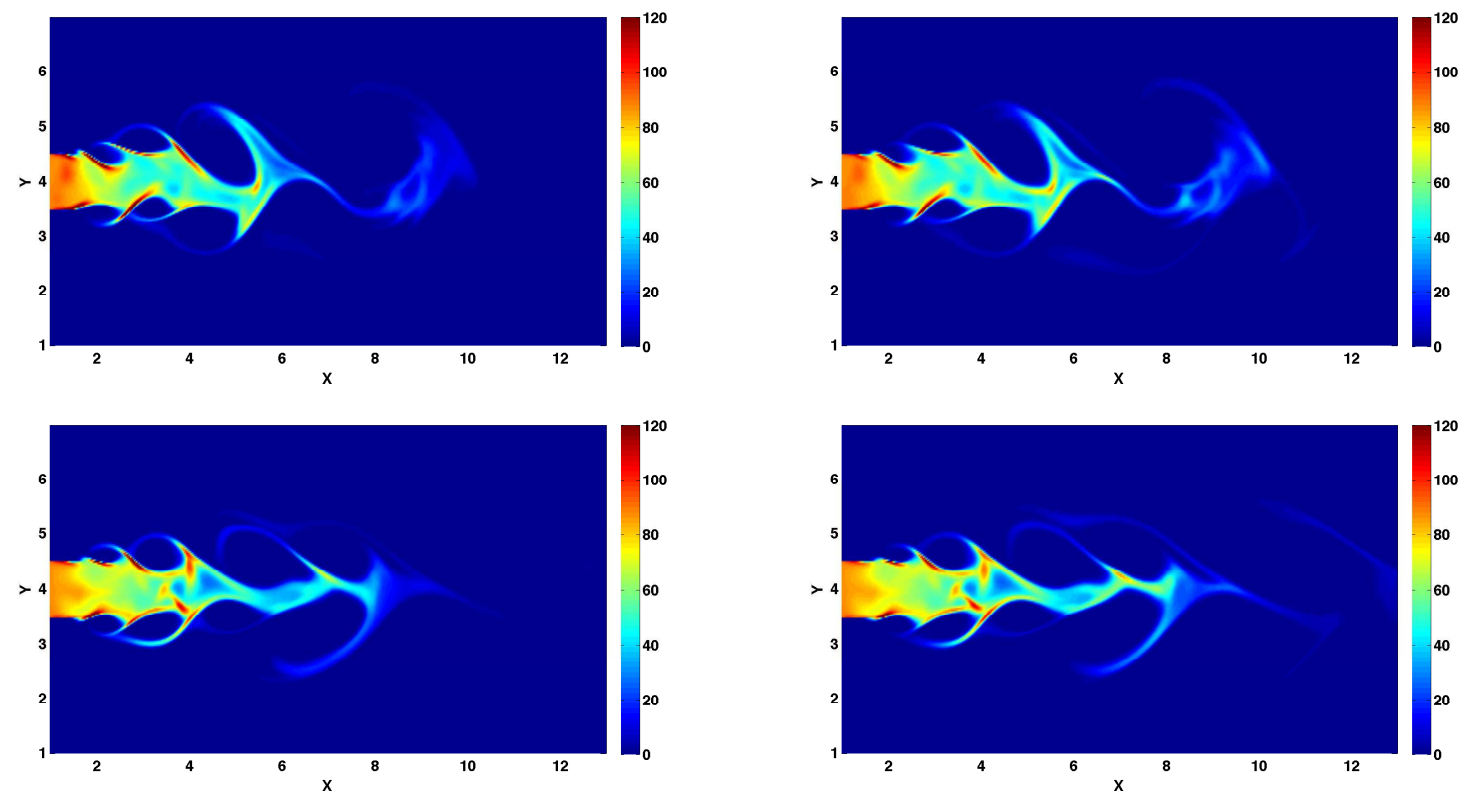

Figure 14: Total mass density of the polydisperse evaporating spray. (Top) Results at time $t=15$. (Bottom) Results at time $t=20$. (Left) EMSM model. (Right) Multi-fluid model with ten sections. The computation is carried out in a $400 \times 200$ cell grid. 

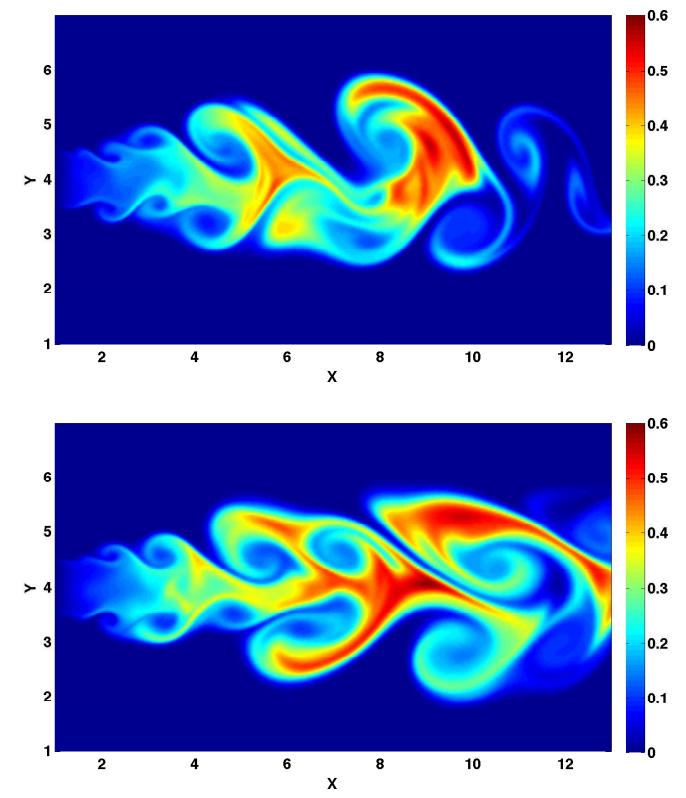
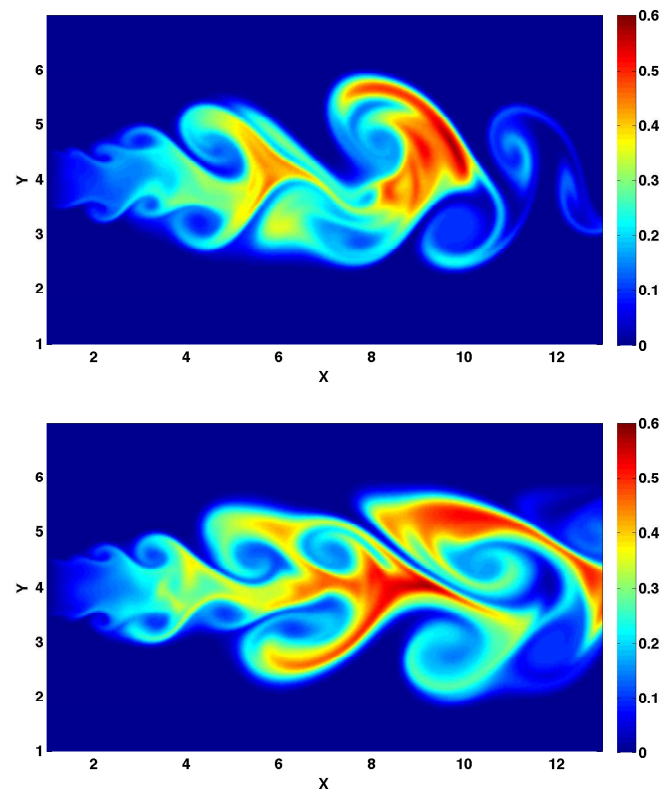

Figure 15: Comparison of the gas-phase fuel mass fraction. (Top) Results at time $t=15$. (Bottom) Results at time $t=20$. (Left) EMSM model. (Right) Multi-fluid model with ten sections. The computation is carried out in a $400 \times 200$ cell grid.

\section{Conclusion}

The aim of this paper was to present a robust scheme which is capable of describing advection and evaporation of high order moments of a size distribution function for both polydisperse aerosols and sprays. This issue is not only limited to aerosols and sprays, but impacts every field where successive integer moment methods are involved. We have proposed a new algorithm for advection which is compatible with an accurate numerical method in order to capture evaporation, is able to automatically preserve the moment space (and thus resolves the usual stability and realizability difficulties), and has second order in space and time on the moments. It can reach comparable levels of accuracy, with a reasonable space discretization, with reference to a Lagrangian simulation, while leading to a much lower level of computational cost compared to the standard multi-fluid approach and can also be extended naturally to unstructured grids. This scheme has been devised using some algebra based on moment theory [12]. The moments are not the quantities which are directly transported. Instead we use the canonical moments which are much easier to control, and allow the moment vector to stay in the moment space by transporting them separately. This method paves the way to better computing and predictivity capacities of Eulerian models with respect to Lagrangian models.

Let us underline that such a method will be especially dedicated to massively parallel computing. The standard Eulerian multi-fluid model has been shown to scale almost perfectly on parallel architecture up to a thousand cores in [22] in the framework of the MUSES3D code developed at EM2C laboratory by S. de Chaisemartin and L. Fréret. The EMSM method has been implemented in MUSES3D as well as in the IFP-C3D code of IFP Enegies nouvelles (unstructured grids, moving meshes - ALE) [26, 28, 29, 1]. The one point that should be improved is the size-velocity correlation which in on its way [47] and the method should be a powerful method in order to capture the dynamics and evaporation of polydisperse sprays on both structured and unstructured meshes. Let us finally underline that the cost of the method will be strongly reduced compared to what we have done so far with Eulerian multi-fluid models on 3D configurations since the number of variable we solve for will be drastically reduced in order to capture poydispersity.

Another point that we should also underline is the fact that we can use a single section as we have done in the present version of the paper, but the method also allows to use several sections with high order moments for each section in the same way as it was proposed in [37] for evaporation. The method with a single section will be very precise as long as the droplet Stokes number range remains below the 
critical Stokes number associated to droplet crossing. For more inertial droplets, as presented in [37], we can consider several size intervals corresponding to similar droplet dynamics, the smaller one being devoted to the droplets with a Stokes number below the critical one. For more inertial droplets, we have to extend the model to droplet crossing trajectory. This work thus enlightens an interesting perspective for the use of Eulerian models in the simulation of high Knudsen number polydisperse sprays, taking advantage of the work done in $[17,10,21,9,27]$. The ground idea is to use a high order moment method for velocity moments combined with quadrature method of moments (QMOM) such as recently done in [51] for droplets with Stokes numbers higher than the critical one. Eventually, a major achievement would be to reach high accuracy in a code, with the dual capability to account for evaporation of sprays, as well as to take into account droplet trajectory crossings for more inertial droplets, while using the method presented in this contribution for the whole size range below the critical Stokes number where a single velocity has been shown to be accurate. This is the subject of our current investigations.

\section{Acknowledgement}

This research was supported by an IFP Energies nouvelles/EM2C CIFRE Ph.D. grant for Damien Kah. The authors would like to thank J. Reveillon and H. Meftah for their help in designing the free jet configuration and for the use of the ASPHODELE Code developed in J. Reveillon's team at CORIA, Rouen, France. The help of S. de Chaisemartin is gratefully acknowledged in performing the numerical simulations of the free jet configuration with the multi-fluid model which he originally designed in his Ph.D. Thesis.

\section{Appendices}

\section{A. Fokker-Planck equation and derivation of semi-kinetic equations for aerosols and sprays}

In the applications targeted in this paper, the droplets evolve through evaporation and interaction with gas molecules which results in transport in physical space, drag and potentially Brownian motion. The kinetic equation verified by the NDF is a Fokker-Planck based equation:

$$
\partial_{t} f+\partial_{\boldsymbol{x}}(\boldsymbol{v} f)-\partial_{S}(R f)=\beta \partial_{\boldsymbol{v}}\left[\left(\left(\boldsymbol{v}-\boldsymbol{u}_{g}\right) f+\frac{\boldsymbol{Q}}{\beta} \partial_{\boldsymbol{v}} f\right)\right]
$$

The second term of the left hand side represents transport in physical space, whereas the third term accounts for the evaporation of the droplets. The terms of the right hand side stand for Stokes drag force and Brownian motion due to the interaction of the droplets with the gas molecules. The evaporation rate, $R$, is the rate of decrease of the droplet surface, $\beta^{-1}$ is the relaxation time of the droplets, $\boldsymbol{u}_{g}$ is the gas velocity, $\mathbf{Q}$ is the matrix of the temporal correlation of accelerations, $\boldsymbol{F}=\left(F_{1}, F_{2}, F_{3}\right)^{t}$ due to Brownian motion in three dimensions, $Q_{i j}(t)=\int_{0}^{\infty} F_{i}(t) F_{j}(t+\tau) d \tau$. We introduce dimensionless variables:

$$
\begin{aligned}
& t^{\prime}=\frac{t U_{0}}{L_{0}}, \quad x^{\prime}=\frac{x}{L_{0}}, \quad S^{\prime}=\frac{S}{S_{0}}, \quad \boldsymbol{v}^{\prime}=\frac{\boldsymbol{v}}{U_{0}}, \quad \boldsymbol{u}_{g}^{\prime}=\frac{\boldsymbol{u}_{g}}{U_{0}}, \quad f^{\prime}=f \frac{U_{0}^{3} L_{0}^{3} S_{0}}{N_{0}}, \\
& \tau_{g}=\frac{L_{0}}{U_{0}}, \quad \mathrm{St}=\frac{U_{0} \beta^{-1}}{L_{0}}, \quad K=\frac{R L_{0}}{S_{0} U_{0}}, \quad \boldsymbol{\sigma}=\frac{\mathbf{Q} \beta^{-1}}{U_{0}^{2}},
\end{aligned}
$$

where $L_{0}$ is a reference length, $U_{0}$ a reference velocity, $S_{0}$ the maximum droplet size, $N_{0}$ a reference number density. The characteristic time of the droplets is $\tau_{g}$, St the Stokes number, $K$ the nondimensional evaporation rate, $\boldsymbol{\sigma}$ the velocity variance at equilibrium of the droplets due to Brownian motion. Written in terms of dimensionless quantities, the Fokker-Planck equation reads:

$$
\partial_{t}^{\prime} f^{\prime}+\partial_{\boldsymbol{x}^{\prime}}\left(\boldsymbol{v}^{\prime} f^{\prime}\right)-\partial_{S}^{\prime}\left(K f^{\prime}\right)=\frac{1}{\mathrm{St}} \partial_{\boldsymbol{v}^{\prime}}\left[\left(\boldsymbol{v}^{\prime}-\boldsymbol{u}_{g}^{\prime}\right) f^{\prime}+\boldsymbol{\sigma} \partial_{\boldsymbol{v}^{\prime}} f^{\prime}\right]
$$

In the following, for the sake of legibility, we will write the dimensionless quantities without the prime sign. Before investigating the system of macroscopic partial differential equation on the size moments which is at the heart of the present contribution, let us first take the moments in velocity conditioned on size and differentiate between two cases, depending on the range of the Stokes number, aerosols for very small Stokes numbers and sprays for moderate Stokes numbers. 
Aerosols

We first consider the aerosol dynamics for which no terms in Eq. (60) can be neglected, since the effect of the collision of the surrounding gas molecules at thermodynamical equilibrium on the aerosol droplets results in a significative velocity variance compared to the slip velocity $\boldsymbol{u}_{g}-\boldsymbol{v}$. As it is classical in statistical physics, a Langevin equation can be associated to the previous Fokker-Planck equation, which in the "viscous limit" or for large times compared to $\beta^{-1}$ when the inertial term in the equation of motion can be disregarded, reduces to a spatial diffusion as studied in the original works of Einstein and Smoluchowski [6]. Since we rather work with NDF and consider the equation (60) as the fundamental model of our droplets, we will obtain the spatially diffusive regime as a singular perturbation using a Chapman-Enskog type of expansion. For the sake of clarity, let us thus rewrite Eq. (60) as :

$$
\partial_{t} f+\partial_{\boldsymbol{x}}(\boldsymbol{v} f)-\partial_{S}(K f)=\frac{1}{\epsilon} J(f), \quad J(f)=\partial_{\boldsymbol{v}}\left[\left(\boldsymbol{v}-\boldsymbol{u}_{g}\right) f+\boldsymbol{\sigma} \partial_{\boldsymbol{v}} f\right] .
$$

The differential operator $J(f)$ appears as a singular perturbation of Eq. (61), in the case $\epsilon \rightarrow 0$. The NDF is solved using a Chapman-Enskog development (see classical references of kinetic theory of gases $[24,16,7,46])$, that is to say that $f$ is decomposed into powers of $\epsilon$ :

$$
f=f^{(0)}+\epsilon f^{(0)} \phi^{(1)}+\epsilon^{2} f^{(0)} \phi^{(2)}+\ldots
$$

where each order of $\epsilon$ is recursively solved from the lower orders of $f$. Moreover, we look at various order perturbations which leave unchanged the macroscopic quantities. In this problem, the sole macroscopic quantity associated to the one-dimensional subspace of invariant of operator $J$ is the total droplet number $\int J(f) \mathrm{d} \boldsymbol{v}=0$, so each $\phi^{(k)}$ verifies $\int f^{(0)} \phi^{(k)}=0$.

The $0^{\text {th }}$ order term of $f, f^{(0)}$, is the solution of $J\left(f^{(0)}\right)=0$, which is the kernel of operator $J$. It can be easily proven that $f^{0}$ is a Maxwell-Boltzmann distribution, which reads:

$$
f^{(0)}=n(t, \boldsymbol{x}, S) \frac{1}{\sqrt{(2 \pi)^{3} \operatorname{det}(\boldsymbol{\sigma})}} \exp \left(-\boldsymbol{C}^{t} \boldsymbol{\sigma}^{-1} \boldsymbol{C}\right), \quad \boldsymbol{C}=\boldsymbol{v}-\boldsymbol{u}_{g} .
$$

The terms of higher order with respect to $\epsilon$ in the Chapman-Enskog expansion (62) satisfy differential equations, the right-hand side of which involve the lower order terms and the kernel of which is associated to the only macroscopic quantity conserved by the $J$ operator, that is total number density of droplets. In this context, we end up with a self-adjoint compact differential operator with a one dimensional kernel in the proper Hilbert space, for which a Fredholm alternative guarantees the resolvability of $\phi^{(1)}$ and leads to the macroscopic equation of order 0 in $\epsilon$ :

$$
\partial_{t} n+\partial_{\boldsymbol{x}}\left(n \boldsymbol{u}_{g}\right)-\partial_{S}(K n)=0
$$

Extending the resolution of Eq.(61) to the first order of $\epsilon$ leads to the following equation:

$$
\partial_{t} n+\partial_{\boldsymbol{x}}\left(n \boldsymbol{u}_{g}\right)-\partial_{S}(K n)=\epsilon \partial_{\boldsymbol{x}}\left(\boldsymbol{\sigma} \partial_{\boldsymbol{x}} n-n\left(D_{t} \boldsymbol{u}_{g}\right)\right), \quad D_{t} \boldsymbol{u}_{g}=\partial_{t} \boldsymbol{u}_{g}+\left(\boldsymbol{u}_{g} . \partial_{\boldsymbol{x}}\right) \boldsymbol{u}_{g}
$$

where the flux at first order in $\epsilon$ delivers a diffusion term as well as an acceleration term which can be related to a normalized pressure gradient in the framework of an incompressible flow for the gaseous phase and which will not be considered in the following for the sake of clarity of the exposition. These assumptions allow to write the Smoluchowski $[23,6]$ equation on the number density conditioned on size:

$$
\partial_{t} n+\partial_{\boldsymbol{x}}\left(\boldsymbol{u}_{g} n\right)-\partial_{S}(K n)=\partial_{\boldsymbol{x}}\left(\boldsymbol{D} \partial_{\boldsymbol{x}} n\right)
$$

where the diffusion operator reads $\boldsymbol{D}=\epsilon \boldsymbol{\sigma}$ and which is valid for time scales large compared to $\beta^{-1}$.

\section{Spray}

In the case of spray droplets, their Stokes regime $(\mathrm{St} \approx 1)$ is such that their dynamics is mainly governed by the drag term, and, in most cases, for the considered time scales, they are not influenced 
by the small scale fluctuations of the Brownian motion. Thus $f$ satisfies the following kinetic equation, called Williams-Boltzmann equation [49]:

$$
\partial_{t} f+\partial_{\boldsymbol{x}}(\boldsymbol{v} f)-\partial_{S}(K f)+\partial_{\boldsymbol{v}}\left(\frac{1}{\mathrm{St}}\left(\boldsymbol{u}_{g}-\boldsymbol{v}\right) f\right)=0
$$

Contrary to the previous case, the drag term is not a singular perturbation in Eq. (67). So an equation on $n$, the number density, but also on $n u=\int f v d v$ which is the droplet mean momentum conditioned on size, have to be derived. These equations are obtained by taking the velocity moment of order 0 and 1 of Eq. (67):

$$
\begin{aligned}
\partial_{t} n+\partial_{\boldsymbol{x}}(n \boldsymbol{u})-\partial_{S}(K n) & =0, \\
\partial_{t}(n \boldsymbol{u})+\partial_{\boldsymbol{x}}(n \boldsymbol{u} \otimes \boldsymbol{u}+n \boldsymbol{P})-\partial_{S}(K n \boldsymbol{u})-n \frac{\boldsymbol{u}_{g}-\boldsymbol{u}}{\mathrm{St}} & =0,
\end{aligned}
$$

where $n \boldsymbol{P}=\int(\boldsymbol{v}-\boldsymbol{u}) \otimes(\boldsymbol{v}-\boldsymbol{u}) f \mathrm{~d} v$ is the droplet pressure tensor.

\section{B. Order accuracy comparison with the scheme proposed in [48]}

The same order accuracy study in Section 5.2 is applied to the scheme designed in [48]. The basic idea is to reconstruct weights and velocity abscissas through the quadrature method detailed in $[17,18]$. While a first order scheme is applied to the abscissas, the weights are reconstructed with $2^{\text {nd }}$ but also more generally $p^{t h}$ order schemes. Reconstructing only weights has the great advantage of ensuring the moments conservation very easily, since the relation between moments and weights is linear. The authors moreover show, on test cases with a constant velocity field, that the numerical order reaches the theoretical one. The temporal integration is performed with an explicit second order Runge-Kutta scheme.

It is possible to adapt this scheme for our study, i.e. size moment advection. Like before, only nodes are reconstructed, size abscissas and velocity values being constant in the cell. Since, for the studied case here, the initial distribution as well as the velocity profiles are not constant, the reconstruction degenerates to first order, while only weight reconstructions are second order. Therefore one expects the transport scheme to have a global order which is closer to one than two. This is confirmed by Table 5 and Fig. 16 and showing the global $L_{1}$ error computation relative to the analytical solution, and the deduced global order given by the slope of the straight line obtained by linear regression from the logarithmic error values. Thus, in terms of pure transport, and for realistic initial profiles, i.e. not assuming that they are constant, the scheme explained in this article is second order in time and space, whereas the scheme designed in [48] degenerates to a first order scheme.

\begin{tabular}{|c|cccc|c|}
\hline Grid size & 25 & 50 & 100 & 200 & Order \\
\hline$m_{0}$ & 0.5745 & 0.3181 & 0.1786 & 0.0959 & 0.86 \\
\hline$m_{1}$ & 0.6452 & 0.3822 & 0.2244 & 0.1258 & 0.78 \\
\hline$m_{2}$ & 0.6556 & 0.3931 & 0.2324 & 0.1312 & 0.77 \\
\hline$m_{3}$ & 0.6589 & 0.3969 & 0.2359 & 0.1336 & 0.77 \\
\hline
\end{tabular}

Table 5: $L_{1}$ error and order of accuracy of the scheme explained in [48] 

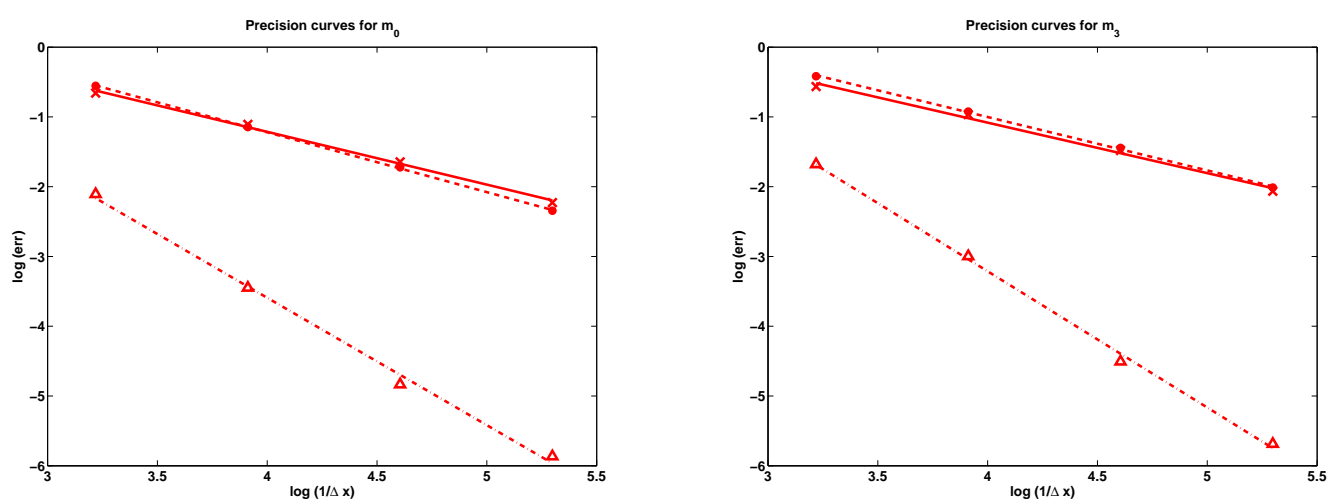

Figure 16: Order accuracy study. Error curves with respect to grid refinement in logarithmic scale. The solid line represents the first order scheme, the dotted-dashed represents the second order scheme, the dashed line represents the scheme of [48]. The symbols represent the logarithm of the error given by the computation. Left: results for $m_{0}$; Right: results for $m_{3}$.

\section{C. Moment vector advection scheme in the context of a non-structured grid}

Although the article focuses on a numerical scheme for the advection of a moment vector in a structured mesh, it is possible, with some adjustments, to extend this scheme to a non-structured mesh based on the ideas developed in the Ph.D. Thesis of G. Dufour [15]. This Appendix gives the principle of a second order scheme for any type of cell geometry. Let $\mathcal{T}$ denote the grid, composed of cells denoted by $K$, delimited by the area $\partial K$. A face of the cell is denoted by $e$, and $\overrightarrow{n_{K, e}}$ is the unitary outwards vector though $e$, as illustrated in Fig 17 . If the moment vector of cell $K$ is $\mathcal{M}_{K}=\left(m_{0, K}, m_{1, K}, m_{2, K}, m_{3, K}\right)^{T}$, then a first order scheme writes:

$$
\mathcal{M}_{K}^{n+1}=\mathcal{M}_{K}^{n}-\frac{\Delta t}{V_{K}} \sum_{e \in \partial K} \Phi\left(\mathcal{M}_{K}^{n}, \mathcal{M}_{K, e}^{n}, \overrightarrow{n_{K, e}}\right)|e|
$$

where $V_{K}$ is the volume of cell $K,|e|$ is the area of the face $e, \mathcal{M}_{K}^{n}$ is the value of the vector $\mathcal{M}$ in cell $K$ at iteration $n$, and $\mathcal{M}_{K, e}^{n}$ is the value of the vector $\mathcal{M}$ of the neighbor cell through the face $e$. The quantity $\Phi\left(\mathcal{M}_{K}^{n}, \mathcal{M}_{K, e}^{n}, \overrightarrow{n_{K, e}}\right)$ is the numerical flux corresponding to the direction of vector $\overrightarrow{n_{K, e}}$. This flux is based on the constant states in cell $K$.
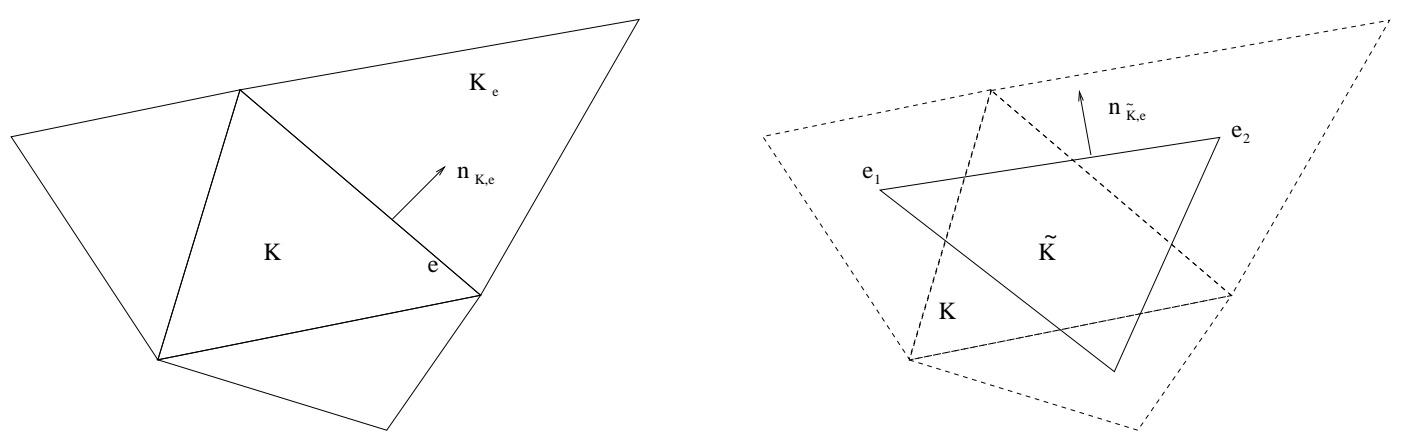

Figure 17: Primary (left) and dual (right) mesh.

For the second order scheme, the vector $\mathcal{W}_{K}=\left(m_{0, K}, p_{1, K}, p_{2, K}, p_{3, K}\right)^{T}$ is considered for linear reconstruction:

$$
\mathcal{W}(x)=\overline{\mathcal{W}_{K}}+\vec{\nabla} \mathcal{W}_{K} \cdot\left(\boldsymbol{x}-\boldsymbol{x}_{K}\right)
$$

where, $\overline{\mathcal{W}_{K}}$ is a modified average value of the quantities computed in order for the scheme to be conservative, as explained in the structured grid case, and $\boldsymbol{x}_{K}$ is the center of cell $K$. The gradient of $\mathcal{W}$, is 
estimated using a Green formula for a cell of the dual mesh (see Fig. (17)-right):

$$
\int_{\tilde{K}} \vec{\nabla} \mathcal{W}_{K} \mathrm{~d} \boldsymbol{x}=\int_{\partial \tilde{K}} \mathcal{W} \cdot \overrightarrow{n_{K, e}} \mathrm{~d} s
$$

Considering that $\vec{\nabla} \mathcal{W}_{K}$ is constant on cell $K$ (because of the linear reconstruction), one gets an estimate of it using a linear interpolation on the face of $\tilde{K}$ through the expression:

$$
\begin{aligned}
V_{K} \vec{\nabla} \mathcal{W}_{K} & =\sum_{e \in \partial K}\left(\int_{0}^{1}(1-\lambda) \mathcal{W}^{n}\left(e_{1}\right)+\lambda W^{n}\left(e_{2}\right) \mathrm{d} \lambda\right)|e| \overrightarrow{n_{K, e}} \\
& =\sum_{e \in \partial K} \frac{1}{2}\left(\mathcal{W}^{n}\left(e_{1}\right)+\mathcal{W}^{n}\left(e_{2}\right)\right)|e| \overrightarrow{n_{K, e}}
\end{aligned}
$$

In order to ensure that $\mathcal{W}_{\min } \leq \mathcal{W}(\boldsymbol{x}) \leq \mathcal{W}_{\max }$ for any $\boldsymbol{x}$ in the cell $K, \vec{\nabla} \mathcal{W}_{K}$ is limited with the following expression:

$$
\widetilde{\vec{\nabla}} \mathcal{W}_{K}=\min \left(1, \frac{\mathcal{W}_{\max }-\mathcal{W}_{K}}{\max _{\boldsymbol{x} \in K}\left|\vec{\nabla} \mathcal{W}_{K} \cdot\left(\boldsymbol{x}-\boldsymbol{x}_{K}\right)\right|}, \frac{\mathcal{W}_{K}-\mathcal{W}_{\min }}{\min _{\boldsymbol{x} \in K}\left|\vec{\nabla} \mathcal{W}_{K} \cdot\left(\boldsymbol{x}-\boldsymbol{x}_{K}\right)\right|}\right) \vec{\nabla} \mathcal{W}_{K}
$$

where $\mathcal{W}_{\text {max }}=\max _{e \in \partial K}\left(\mathcal{W}_{K_{e}}\right)$ is the maximum value of $\mathcal{W}$ in the cell $K$, and $\mathcal{W}_{\min }=\min _{e \in \partial K}\left(\mathcal{W}_{K_{e}}\right)$ is the minimum value, $\mathcal{W}_{K_{e}}$ denoting the value of the vector $\mathcal{W}$ on the edge of the cell $K$. Then, the value of $\mathcal{W}_{e, K}$, taken at the center of the face $e$ is assessed through:

$$
\mathcal{W}_{e, K}=\overline{\mathcal{W}_{K}}+\vec{\nabla} \mathcal{W}_{K} \cdot\left(\boldsymbol{x}_{e}-\boldsymbol{x}_{K}\right)
$$

The computation of the bar values follows the same logic as in the structured case, that is to say that the mean value in cell $K$ of the moments expressed as a function of the canonical moments must be equal to the mean value of the moments.

Given this reconstruction with this slope limiter, the maximum principle for the canonical moments is satisfied and the moment space is preserved throughout the whole cell $K$.

The temporal integration is performed using an explicit second order Runge-Kutta method. In this context, the solution reads:

$$
\mathcal{M}_{K}^{n+1}=\frac{1}{2} \mathcal{M}_{K}^{n}+\frac{1}{2} \widetilde{\mathcal{M}}_{K}^{n+1}-\frac{\Delta t}{2 V_{K}} \sum_{e \in \partial K} \Phi\left(\widetilde{\mathcal{M}}_{K}^{n+1}, \widetilde{\mathcal{M}}_{K, e}^{n+1}, \overrightarrow{n_{K, e}}\right)|e|
$$

where

$$
\widetilde{\mathcal{M}}_{K}^{n+1}=\mathcal{M}_{K}^{n}-\frac{\Delta t}{V_{K}} \sum_{e \in \partial K} \Phi\left(\mathcal{M}_{K}^{n}, \mathcal{M}_{K, e}^{n}, \overrightarrow{n_{K, e}}\right)|e|
$$

As it is classical for non-structured grids, restrictions on the CFL number apply. The maximum possible CFL value enforcing the scheme properties (positivity of density, of the moments and preservation of the moment space) depends on the considered mesh geometry. It can be proven that this scheme is second order in time and space since the spatial reconstruction and time integration are both second order accurate.

\section{References}

[1] G. A. BIRD, Ifp-c3d: An unstructured parallel solver for reactive compressible gas flow with spray, Oxford Science Publications, 42 (1994).

[2] F. Bouchut, On zero pressure gas dynamics, in Advances in kinetic theory and computing, vol. 22 of Ser. Adv. Math. Appl. Sci., World Sci. Publ., River Edge, NJ, 1994, pp. 171-190. 
[3] F. Bouchut, S. JIN, AND X. LI, Numerical approximations of pressureless and isothermal gas dynamics, SIAM J. Numer. Anal., 41 (2003), pp. 135-158.

[4] Y. Brenier AND E. GREnIER, Sticky particles and scalar conservation laws, SIAM Journal of Numerical Analysis, 35 (1998), pp. 2317-2328.

[5] C. Chalons, D. Kah, And M. Massot, Beyond pressureless gas dynamics: quadrature-based velocity moment models, Communication in Mathematical Sciences (accepted for publication, in revision), (2011), pp. 1-21. available online at http://hal.archives-ouvertes.fr/hal-00535782/en/ and at arXiv:1011.2974v1 [math.NA].

[6] S. Chandrasekhar, Stochastic problems in physics and astronomy, Rev. Mod. Phys., 15 (1943), pp. 1-89.

[7] S. Chapman and T. G. Cowling, The mathematical theory of nonuniform gases, Cambridge Mathematical Library, Cambridge University Press, Cambridge, third ed., 1990.

[8] S. De Chaisemartin, Eulerian models and numerical simulation of turbulent dispersion for polydisperse evaporating sprays, $\mathrm{PhD}$ thesis, Ecole Centrale Paris, France, 2009. Available online at http://tel.archives-ouvertes.fr/tel-00443982/en/.

[9] S. de Chaisemartin, L. Fréret, D. Kah, F. Laurent, R. Fox, J. Reveillon, and M. MAssot, Eulerian models for turbulent spray combustion with polydispersity and droplet crossing, Comptes Rendus Mécanique, 337 (2009), pp. 438-448. (Special Issue Combustion for Aerospace Propulsion).

[10] S. de Chaisemartin, L. Fréret, D. Kah, F. Laurent, R. O. Fox, J. Reveillon, And M. MASSOT, Eulerian models for turbulent spray combustion with polydispersity and droplet crossing: modeling and validation, Proceedings of the Summer Program 2008, (2009), pp. 265-276.

[11] O. Desjardin, R. Fox, And P. Villedieu, A quadrature-based moment method for dilute fluidparticle flows, J. Comput. Phys., 227 (2008), pp. 2514-2539.

[12] H. Dette And W. J. Studden, The theory of canonical moments with applications in statistics, probability, and analysis, Wiley Series in Probability and Statistics: Applied Probability and Statistics, John Wiley \& Sons Inc., New York, 1997. A Wiley-Interscience Publication.

[13] F. Doisneau, F. Laurent, A. Muronne, J. Dupays, and M. Massot, Optimal eulerian model for the simulation of dynamics and coalescence of alumina particles in solid propellant combustion, in Proceedings of the International Conference on Multiphase Flows, Tampa, Florida, 2010, pp. 1-15. available online at http://hal.archives-ouvertes.fr/hal-00498215/en/.

[14] F. Doisneau, F. Laurent, A. Murrone, J. Dupays, and M. Massot, Evaluation of Eulerian Multi-Fluid models for the simulation of dynamics and coalescence of particles in solid propellant combustion, Journal of Computational Physics (submitted), (2011).

[15] G. Dufour, Modélisation multi-fluide eulérienne pour les écoulements diphasiques à inclusions dispersées, PhD thesis, Université Paul Sabatier Toulouse III, 2005.

[16] J. H. Ferziger And H. G. KAPER, Mathematical Theory of transport processes in gases, NorthHolland, Amsterdam, 1972.

[17] R. O. Fox, A quadrature-based third-order moment method for dilute gas-particle flow, J. Comput. Phys., 227 (2008), pp. 6313-6350.

[18] _ Optimal moment sets for multivariate direct quadrature methods of moments, Industrial and Engineering Chemistry Research, 48 (2009), pp. 6313-6350. 
[19] R. O. Fox, F. LAURENT, AND M. MAssot, Numerical simulation of spray coalescence in an eulerian framework: direct quadrature method of moments and multi-fluid method, Journal of Computational Physics, 227 (2008), pp. 3058-3088.

[20] L. Fréret, S. De Chaisemartin, J. Reveillon, F. Laurent, And M. Massot, Eulerian models and three-dimensional numerical simulation of polydisperse sprays, in Proceedings of the International Conference on Multiphase Flows, Tampa, Florida, 2010, pp. 1-12. Available online at http://hal.archives-ouvertes.fr/hal-00498207/en/.

[21] L. Fréret, F. Laurent, S. de Chaisemartin, D. Kah, F. Laurent, P. Vedula, R. O. Fox, O. Thomine, J. Reveillon, And M. Massot, Eulerian moment models for polydisperse weakly collisional sprays: model and validation, in Proceedings of the Summer Program 2008, Center for Turbulence Research, Stanford University, 2009, pp. 277-288.

[22] L. Fréret, O. Thomine, J. Reveillon, S. de Chaisemartin, F. Laurent, and M. Massot, On the role of preferential segregation in flame dynamics in polydisperse evaporating sprays, in Proceedings of the Summer Program 2010, Center for Turbulence Research, Stanford University, 2011, pp. 383-392.

[23] S. K. Friedlander, Smoke, Dust, and Haze, Fundamental of Aerosol Dynamics, Topics in Chemical Engineering, Oxford University Press, second ed., 2000.

[24] H. Grad, Principles of the kinetic theory of gases, in Handbuch der Physik (herausgegeben von S. Flügge), Bd. 12, Thermodynamik der Gase, Springer-Verlag, Berlin, 1958, pp. 205-294.

[25] J. Greenberg, I. Silverman, And Y. Tambour, On the origin of spray sectional conservation equations, Combustion and Flame, 93 (1993), pp. 90-96.

[26] D. KAH, Taking into account polydispersity for the modeling of liquid fuel injection in internal combustion engines, PhD thesis, Ecole Centrale Paris, 2010.

[27] D. Kah, F. Laurent, L. Fréret, S. de Chaisemartin, R. Fox, J. Reveillon, and M. MasSOT, Eulerian quadrature-based moment models for polydisperse evaporating sprays, Flow, Turbulence and Combustion, 85 (2010), pp. 649-676. (Special Issue Dedicated to Stephen B. Pope).

[28] D. KAh, F. LAURent, M. Massot, And S. JAY, Modeling of polydisperse sprays using a high order size moment method for the numerical simulation of advection and evaporation, in Proceedings of the 11th ICLASS, International Conference on Liquid Atomization and Spray Systems Vail, Colorado, 2009.

[29] D. Kah, M. Massot, Q. Tran, S. Jay, And F. Laurent, A high order moment method with mesh movement for the description of a polydisperse evaporating spray, in Proceedings of the International Conference on Multiphase Flows, Tampa, Florida, 2010. available online at http://hal.archivesouvertes.fr/hal-00498214/en/.

[30] M. Klein, A. SAdiki, AND J. JAniCKA, A digital filter based generation of inflow data for spatially developing direct numerical or large eddy simulations, J. Comput. Phys., 186 (2003), pp. 652-665.

[31] F. LAURENT, Numerical analysis of Eulerian multi-fluid models in the context of kinetic formulations for dilute evaporating sprays, M2AN Math. Model. Numer. Anal., 40 (2006), pp. 431-468.

[32] F. Laurent and M. Massot, Multi-fluid modeling of laminar poly-dispersed spray flames: origin, assumptions and comparison of the sectional and sampling methods, Combust. Theory and Modelling, 5 (2001), pp. 537-572.

[33] R. J. LeVEque, Finite volume methods for hyperbolic problems, Cambridge Texts in Applied Mathematics, Cambridge University Press, Cambridge, 2002.

[34] D. L. Marchisio And R. O. Fox, Solution of population balance equations using the direct quadrature method of moments, Journal of Aerosol Science, 36 (2005), pp. 43-73. 
[35] M. MASsOt, Eulerian multi-fluid models for polydisperse evaporating sprays, in Computational Models for Turbulent Multiphase Reacting Flows, vol. 492 of CISM Courses and Lectures, SpringerWienNewYork, Vienna, 2007, pp. 79-123. Editors D.L. Marchisio and R. O. Fox, Udine, July 2006.

[36] M. Massot, F. Laurent, S. De Chaisemartin, L. Fréret, and D. Kah, Eulerian multi-fluid models: modeling and numerical methods, in Modelling and Computation of Nanoparticles in Fluid Flows, Lectures Notes of the von Karman Institute, NATO RTO-EN-AVT-169, 2009, pp. 1-86. Available online at http://www.rta.nato.int/Pubs/RDP.asp?RDP=RTO-EN-AVT-169.

[37] M. Massot, F. Laurent, D. Kah, and S. De Chaisemartin, A robust moment method for evaluation of the disappearance rate of evaporating sprays, SIAM J. Appl. Math., 70 (2010), pp. 32033234.

[38] R. MCGRAw, Numerical advection of correlated tracers: preserving particle size/composition moment sequences during transport of aerosol mixtures, J. Phys.: Conf. Ser., 78 (2007).

[39] L. R. Mead and N. Papanicolaou, Maximum entropy in the problem of moments, J. Math. Phys., 25 (1984), pp. 2404-2417.

[40] H. Meftah, Simulation Numérique Directe d'un spray en évaporation : Analyse et modélisation du mélange turbulent et des transferts thermiques, PhD thesis, INSA de Rouen, France, 2008.

[41] J.-B. Mossa, Extension polydisperse pour la description euler-euler des écoulements diphasiques réactifs - TH/CFD/05/74, PhD thesis, Institut National Polytechnique de Toulouse, 2005.

[42] D. Ramkrishna And A. G. Fredrickson, Population Balances: Theory and Applications to Particulate Systems in Engineering, Academic Press, 2000.

[43] J. REveillon, DNS of spray combustion, dispersion evaporation and combustion, in Computational Models for Turbulent Multiphase Reacting Flows, vol. 492 of CISM Courses and Lectures, SpringerWienNewYork, Vienna, 2007, p. 229. Editors D.L. Marchisio and R. O. Fox, Udine, July 2006.

[44] J. Reveillon And F. Demoulin, Effects of the preferential segregation of droplets on evaporation and turbulent mixing, Journal of Fluid Mechanics, 583 (2007), pp. 273-302.

[45] G. Strang, On the construction and comparison of difference schemes, SIAM Journal of Numerical Analysis, 5 (1968), pp. 506-517.

[46] H. Struchtrup, Macroscopic transport equations for rarefied gas flows, Interaction of Mechanics and Mathematics, Springer, Berlin, 2005. Approximation methods in kinetic theory.

[47] A. Vié, F. Laurent, And M. Massot, Size-velocity correlations in high order moment methods: modelling and numerical issues, Journal of Computational Physics (submitted), (2011).

[48] V. Vikas, Z. Wang, A. Passalacqua, and R. O. Fox, Realizable high-order finite-volume schemes for quadrature-based moment methods, Journal of Computational Physics, 230 (2011), pp. $5328-5352$.

[49] F. A. Williams, Spray combustion and atomization, Phys. Fluids, 1 (1958), pp. 541-545.

[50] D. L. Wright, Numerical advection of moments of the particle size distribution in Eulerian models, Journal of Aerosol Science, 38 (2007), pp. 352-369.

[51] C. YuAn AND R. Fox, Conditional quadrature method of moments for kinetic equations, Journal of Computational Physics, In Press, Corrected Proof (2011), pp. -. 